\title{
Nanoparticle formation and dusty plasma effects in DC sputtering discharge with graphite cathode.
}

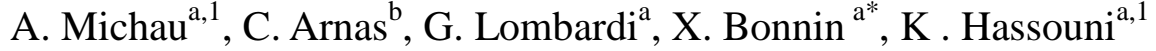 \\ ${ }^{a}$ LSPM, CNRS, 99 Avenue J. B. Clément, 93430 Villetaneuse, France \\ ${ }^{b}$ Laboratoire PIIM, CNRS, Aix-Marseille université, Centre Saint-Jérôme, 13397 Marseille, France
}

\begin{abstract}
We developed a model for the nucleation, growth and transport of carbon dust particles in a DC discharge. The carbon source comes from the sputtering of a graphite cathode resulting in the production of primary clusters and then of nanoparticles. We consider the ionic cluster growth as well as the particle growth and charging and the influence of both on the discharge equilibrium. We found that the discharge becomes electronegative for long duration when particle density reaches $10^{9} \mathrm{~cm}^{-3}$ and particle size $45 \mathrm{~nm}$. The corresponding transition modifies the electric field profile in the vicinity of the field reversal region in the negative glow. We then analyze the space and time evolution of the different discharge characteristics and the mechanisms involved in the discharge. We showed that particle density is governed by nucleation, coagulation and transport, while particle size is mainly governed by the deposition of the small neural clusters emitted at the cathode on the particle surface
\end{abstract}

Key-words : DC discharges, dusty plasma, cluster, model

*Present address : ITER Organization, Building 72/4005, POP, Science Division Route de Vinon-sur-Verdon 13067 St Paul Lez Durance Cedex - France

${ }^{1}$ corresponding author : armelle.michau@1spm.cnrs.fr, khaled.hassouni@1spm.crs.fr 


\section{Introduction}

Although, dust formation was experimentally observed in DC discharges in the early 90's [1],[2], there are very few studies, especially through modeling, of this phenomenon [3] which was mainly considered for capacitively coupled parallel plate RF (CCPP-RF) discharges in silane and, to a lesser extent, in acetylene [4]. In these discharges the molecular species present in all the plasma volume give rise to a molecular growth and a subsequent solid particle-nucleation. These conditions are very different from those encountered when particle formation results from cathode sputtering as experimentally observed in rare gas DC or DCmagnetron discharges [1]. In these last cases, the growth precursors are generated at the cathode and therefore sputtering kinetics is one of the key phenomena that govern particle formation and growth. Further, the discharge structure and dynamic are different from the CCPP-RF configuration. As a result, one may expect that the driving mechanisms for particle formation and growth and the subsequent dusty plasma effect would be different from those encountered in CCPP-RF dusty plasmas.

In this work, we are interested in the study of the mechanisms involved in particle formation through sputtering of a graphite electrode in DC discharges. Our purpose is to understand how carbon particles observed in this kind of discharges [5], [6] are produced, and to investigate the changes in the discharge characteristics, density, electric field, etc., when particle formation is significant enough to induce a dusty plasma effect.

A scenario of particle formation and growth was previously proposed [7], in which primary carbon species $\mathrm{C}$, and to a lesser extent, $\mathrm{C}_{2}$ and $\mathrm{C}_{3}$, are produced as a result of the sputtering of the graphite cathode by argon ion and fast neutral species. These primary species undergo molecular growth that result in the formation of larger clusters, which-may end up with nucleation of solid particles, if the discharge conditions are appropriate. The particle population and charge evolve under the effect of aerosol dynamic phenomena, transport, surface-growth and particle-coagulation.

Based on this scenario, we have previously developed a numerical model that enables us investigating molecular growth and particle nucleation in DC sputtering discharges for conditions where the density of clusters and carbonaceous particle is small enough so as the discharge equilibrium is not affected by clustering $[8,9]$. In these conditions we showed in particular, the key-role of the field reversal that occurs in the negative glow of the discharge [7]. This effect induces a trapping of the negative clusters, the residence time of which becomes long enough to allow for efficient molecular growth.

A question that naturally arises is whether this very weak field reversal still plays a key-role when dusty plasma effect, i.e., coupling between discharge, particles and clusters dynamics, becomes significant and should be taken into account.

As a matter of a fact, experimental measurements showed that carbon particles are obtained at a density value as high as $10^{8} \mathrm{~cm}^{-3}[10]$. Taking into account the typical electron density in this discharge, i.e., between $10^{8}$ and $10^{10} \mathrm{~cm}^{-3}$ depending on the discharge region, and the large charge number that could be carried-out by submicron particles, the contribution of solid particles to the discharge equilibrium and therefore the dusty plasma effect should be significant.

In this paper, we specifically address this question and examine clustering, nucleation and particle growth while taking into account a dusty plasma effect that would result from the coupling between molecular growth kinetics and solid particle dynamics on one hand, and the discharge equilibrium on the other hand. We propose to investigate how this coupling affects the growth kinetics and the discharge characteristics. Especially, one may expect that the presence of heavy negative particles at a significant density would affect the space charge field and the trapping efficiency. 
For this purpose, we substantially improved the model that was previously developed to investigate the molecular growth of carbon clusters and the onset of particle formation $[8,9]$ in order to achieve a self-consistent description of clustering, aerosol dynamic and discharge equilibrium.

The paper includes five sections. In section 2, we briefly describe the experimental system considered in this work. The details of the model used to describe the dusty plasma are discussed in section 3 . The results are presented and discussed in section 4 . The main conclusions that arise from this work are summarized in section 5.

\section{Investigated Discharges}

We consider argon DC glow discharges, generated between two parallel electrodes of $10 \mathrm{~cm}$ diameter [6]. The cathode is in polycrystalline graphite and the grounded anode, in stainless steel. The distance between the electrodes is $14 \mathrm{~cm}$. The argon pressure is set to $60 \mathrm{~Pa}$ and the discharge current is set to a constant value of $80 \mathrm{~mA}$. In such conditions, the discharge voltage is a free parameter and the cathode takes spontaneously a bias value of $\sim-550 \mathrm{~V}$. As a consequence, the cathode is sputtered by ions and by fast argon atoms that are produced through charge exchange in the cathode sheath.

A detailed experimental study was performed on the discharge described above [5], which clearly indicates that the sputtering of the graphite cathode results indeed in the injection of carbon species, probably carbon atoms and, to a lesser extent, small $\mathrm{C}_{2}$ and $\mathrm{C}_{3}$ molecules, in the discharge. Langmuir probe measurements at $300 \mathrm{~s}$ showed that the electron density is approximately $10^{10} \mathrm{~cm}^{-3}$ in the negative glow (NG). The dust particles collected on the anode showed an average diameter of $\sim 40 \mathrm{~nm}$, while the average dust density was estimated to be around $2.10^{8} \mathrm{~cm}^{-3}$ [10]. A simple estimation from the Orbital Motion Limited (OML) theory shows that these dust particles are negatively charged and can carry up to 10 elementary charges. The total density of the charge carried out by the solid particles and the negatively charged clusters may exceed, at least in some discharge regions, the charge density corresponding to electrons. As a result, a transition to an electronegative discharge is expected, which would result in a significant change in the electric structure of the discharge. The confinement and charging process of dust particles would be modified due to the strong coupling between aerosol dynamic and discharge equilibrium. In the next section, we present the dusty plasma effects in the discharge of interest by taking into account the coupling between the discharge equilibrium, the growth of negative clusters and the aerosol dynamics in a self-consistent way. The model develop in this aim is discussed in the next section.

\section{A dusty DC discharge model}

\section{II.1. Phenomenological description}

The model used in this work is based with a large number of improvements on the one described in [7]. In particular, the present model takes into account the coupling between molecular growth and dust particle aerosol dynamic on one hand, and the discharge equilibrium on the other hand. As a result, this model enables us describing properly the dusty plasma effect, while our previous model [7] only describes molecular growth and particle formation assuming pristine argon discharge conditions.

In this model, although it is not mandatory, we assume a short discharge, i.e., no positive column. We distinguish three regions in the discharge vessel: the cathode sheath (CS), the 
negative glow (NG) and the Faraday dark space (FDS). The cathode sheath region is mainly considered because it provides the fast secondary electrons that maintain the discharge in the negative glow. The molecular growth of carbon clusters is assumed to take place in the NG and FDS through a collisional model that involves both neutral and negative clusters. Nucleation and growth of solid carbonaceous particles are also assumed to take place in the NG and the FDS. The model takes into account the field reversal effect in the NG which induces the trapping of the negatively charged species and solid particles.

As a result, two main modules have been developed. The first one combines a semi-analytical model and a Monte Carlo simulation to determine the sheath characteristics and the non local ionization source terms in the NG and the FDS. The second module describes the coupled phenomena of (i) transport of the discharge species, i.e. argon-ion, charged and neutral carbon clusters, (ii) molecular growth of carbon clusters, and (iii) nucleation, growth and transport of solid carbon particles (dust) in the FDS and the NG. This module combines several submodules that allow determining: the electric field structure in the NG and the FDS, the cluster densities, the nucleation rate and the solid particle population density, charge and size.

In the next section we describe the models used in these different sub-modules.

\section{II.2. Sheath, non local ionization and electron population models.}

The sheath characteristics are determined from the well known classical sheath model described for example in [11]. For a given background gas pressure and plasma density in the $\mathrm{NG}$, the sheath thickness and the secondary emission coefficient are determined from the current balance equation and Poisson's equation assuming a matrix-sheath. Then, once the sheath characteristics, i.e., essentially the thickness and secondary emission coefficient, are known, a Monte Carlo simulation of electron kinetics in the gap region, i.e., sheath, NG and FDS in the case of short discharges, is performed. These simulations yield the rate of non local ionization needed to express charged species balance in the NG region.

Electron kinetics in this quite well known DC discharge is surprisingly complex [12]. Basically, three electron groups may be distinguished in the discharge: (i) the fast electrons, with energy above the ionisation threshold, that are produced in the sheath and induce the non local ionization in the NG and are no more present in the FDS, (ii)the intermediate energy electrons that results from fast electrons that loose their energy and end up with energy below the first excitation threshold, (iii) the cold electron that are produced through the ionization processes that take place in the NG and that show very low energy, much below the first ionization threshold.

The fast and intermediate electrons show very weak populations and insure ionization processes and current continuity, respectively. The population of cold electron that are present in much larger number insure the space charge and one may therefore neglect the population of fast and intermediate electrons in determining the E-field in the NG and FDS.

\section{II.3. Collisional processes and transport phenomena in the dusty NG and FDS}

\section{II.3.a. Molecular growth and transport of Carbon clusters}

As mentioned above, some aspects of this model was discussed in [7]. Therefore, we restrict ourselves here to the description of the key assumptions and the main peculiarities of the model, and especially the characteristics that are related to the dusty plasma effect.

The model assumes that cluster formation is due to the sputtering of the graphite cathode by energetic argon ions, which produces carbon as $\mathrm{C}, \mathrm{C}_{2}$ and $\mathrm{C}_{3}$ [13]. These clusters are created at the carbon cathode and are subsequently transported inside the sheath, the NG and the FDS 
where they experience collisions with electrons, positive argon ions (charge transfer) and other clusters (molecular growth). These collisions result in ionization, attachment/detachment processes, charge transfer and molecular growth. As a result, a complex plasma containing a cluster population with a large size distribution is formed.

Positively charged carbon species created by neutral clusters ionisation or charge transfer with positive argon ion have extremely short a residence time of approximately $10^{-6} \mathrm{~s}$, in the region located between the cathode and the field reversal position. This time is much shorter than the characteristic time of molecular growth and sticking on the surface of negatively charged particles. These were estimated to be of the order of $1 \mathrm{~s}$ and $10^{-2} \mathrm{~s}$, respectively. The positive species created in this region are therefore lost at the graphite cathode before contributing to molecular growth and particle growth through surface deposition. In the region located beyond the field reversal location, the carbon cluster ionization is fairly slow with a characteristic time of approximatively $10 \mathrm{~s}$. Therefore the contribution of positively charged clusters to molecular growth and sticking to particles is limited by the ionization kinetics. This has to be compared to the characteristic time of particle growth through neutral cluster deposition which is approximately $10^{-4} \mathrm{~s}$, indicating that particle growth through sticking is dominated by neutral clusters in this region.

The above analysis shows that the contribution of positive ions to the molecular growth and aerosol dynamic is negligible in the whole investigated discharge system. These species were therefore not considered in our model.

On the opposite, collisions with electron produce negative clusters that can be trapped in the positive potential of the discharge. Although both singly and doubly charged negative carbon clusters can be formed, doubly charged negative clusters usually show population much smaller than singly charged negative clusters and are not taken into account [7]. Consequently, we only consider neutral and single charge negative clusters. Further, the model takes into account the existence of different conformation, i.e., linear, cyclic, bicyclic, etc., for each cluster size [14]. We describe the formation of large clusters from the $\mathrm{C}_{1} \mathrm{C}_{2}$ and $\mathrm{C}_{3}$ species emitted from the cathode through a collisional scheme that involves:

- Molecular aggregation for neutral and charged clusters

$$
\begin{array}{r}
\mathrm{C}_{\mathrm{x}}+\mathrm{C}_{\mathrm{n}}->\mathrm{C}_{\mathrm{n}+\mathrm{x}} \\
\mathrm{C}_{\mathrm{x}}+\mathrm{C}_{\mathrm{n}}{ }^{-} \rightarrow>\mathrm{C}_{\mathrm{n}+\mathrm{x}}{ }^{-}
\end{array}
$$

- Charge transfer

$$
\mathrm{C}_{\mathrm{n}}^{-}+\mathrm{C}_{\mathrm{m}}->\mathrm{C}_{\mathrm{n}+} \mathrm{C}_{\mathrm{m}}^{-}
$$

- Electron Attachment/Detachment

$$
\begin{gathered}
\mathrm{C}_{\mathrm{n}}+\mathrm{e}^{-}->\mathrm{C}_{\mathrm{n}}^{-} \\
\mathrm{e}^{-}+\mathrm{C}_{\mathrm{n}}->\mathrm{C}_{n}+2 \mathrm{e}^{-}
\end{gathered}
$$

where $\mathrm{x}=1-3, \mathrm{n}>1, \mathrm{~m}>1$

As may be noticed, we did not use a reversible molecular growth model. We indeed assume that large clusters cannot undergo fragmentation to produce more stable smaller clusters. Nevertheless, the molecular model we are using does take into account the existence of magic numbers in the cluster size distribution and therefore predict much higher abundance for clusters corresponding to these magic numbers (such as fullerenes).

The estimation of the rate constants for the processes (R1-R5) is thoroughly discussed in [7]. To take into account the existence of magic numbers in the size distribution, the rate constants include a correction that depends on the formation enthalpy and the subsequent relative thermodynamic stability of the different clusters. Of course more accurate description of 
cluster size distribution would require taking into account fragmentation (reversibility). However this would make the molecular growth model far more complex.

The molecular growth mechanism described by processes (R1-R5) is assumed to take place up to a maximum size that defines the largest molecular edifice (LME). Aggregation processes involving this LME are assumed to lead to the nucleation of a solid particle.

In the present study, we choose a LME of 30-atom carbon clusters which leads, taking into account the existence of a negative and a neutral clusters and several isomers for each size, to a cluster system of 126 species.

Once solid particles are formed, carbon cluster may also undergo heterogeneous chemistry on the surface of the particles. We assume in the present model that this heterogeneous chemistry essentially consists of sticking reactions that results in a depletion of cluster population and is therefore described using a phenomenological sticking coefficient.

As far as transport effects in the NG and FDS regions are concerned, the neutral species are transported by diffusion while the charged species are transported by drift and diffusion in the very weak electric field.

We are mainly interested in the trapping mechanism that confine negative clusters and dust particle in the axial direction between the sheath and the FDS. Consequently, only axial distribution of species densities and electric field between the sheath edge and the anode surface are of interest in the present study. Therefore, we make use of a radially averaged 1D model where the species losses at the reactor wall are described through a macroscopic source term. As a result, the time evolution for the density of a cluster of size ' $i$ ' with a charge ' $z$ ' is governed by the continuity equation:

$$
\frac{\partial n_{i}^{z}}{\partial t}=-\nabla\left(-D_{i} \vec{\nabla} n_{i}^{z}+\mu_{i} \cdot z \cdot \vec{E}\right)+W_{i}^{z}-P_{i}^{p}-P_{i}^{r} ; \text { with } \mathrm{i}=1,30 \mathrm{z}=-1,0
$$

Equation (1) expresses the balance between the time evolution of the cluster density and the rates of change due to the drift-diffusion transport (first term in the right hand side) and to the source terms related to molecular growth processes (second term in the RHS), heterogeneous sticking on solid particles (third term in the RH) and on the reactor wall (last term).

$n_{i}^{z}, D_{i}, \mu_{i}, W_{i}^{z}$ represent the density, diffusion coefficient, mobility and net production rate through gas phase processes of cluster ' $i$ ' of charge $z$ respectively.

Drift and diffusion are assumed to take place in argon that remains the major species. Diffusion coefficients are determined using a Lenard-Jones potential, while mobility coefficients are obtained from Einstein relation.

The net production rate $W_{i}^{z}$ takes into account the processes $\mathrm{R} 1-\mathrm{R} 5$, the rate constants of which are thoroughly discussed in [9] and references therein.

$P_{i}^{p}$ and $P_{i}^{r}$ represent the loss rate of cluster $i$ through heterogeneous processes at the surface of carbon particles and at the reactor wall. Note that since we do not consider positively charged clusters and multiply charged negative clusters, the value of $z$ may be either 0 or -1 in the present model. $E$ is the electric field.

The solution of the continuity equations (1) requires specifying boundary conditions at the cathode and anode surface. The boundary conditions for the small $\mathrm{C}, \mathrm{C}_{2}$ and $\mathrm{C}_{3}$ are derived from the sputtering rate of the graphite cathode assuming that $\mathrm{C}, \mathrm{C}_{2}$ and $\mathrm{C}_{3}$ represents $70 \%$, $20 \%$ and $10 \%$ of the sputtered carbon. We assumed that all the other neutral cluster sticks to the cathode surface with a sticking coefficient of 1 . As for the negative clusters, we assume a zero density at the cathode surface since they experience the repulsion of the potential.

At the anode surface, the boundary conditions express the balance between drift-diffusion fluxes and surface reactions, i.e., sticking at the surface. 


\section{II.3.b. Nucleation, growth, charging and transport of solid particles}

As mentioned previously, aggregation processes involving this LME are assumed to lead to the nucleation of a solid particle that may undergo an aerosol dynamic with transport phenomena and growth processes through surface sticking of clusters or particle coagulation processes. Fragmentation processes are not taken into account.

In principle, describing the growth and charging of solid particles requires considering detailed size and charge distributions of the solid particle population [15]. Coupling such a detailed aerosol dynamic to the detailed model of molecular growth and to the discharge equilibrium model would however result in too much complexity and can make the problem intractable. Therefore, we made the choice in this work to simplify the description of the aerosol dynamic, while keeping a detailed description of the molecular growth, i.e., the nucleation step, and the coupling between cluster kinetics, solid particle growth and discharge equilibrium.

As a result the particle population is described through three physical parameters: the particle density, the particle volume mean diameter and the particle averaged charge.

The particle density is governed by the following transport equation that expresses the balance between the rates of change in particle number density due to transport fluxes, particle nucleation and coagulation process :

$$
\frac{\partial n_{p}}{\partial t}=-\vec{\nabla}\left(\vec{F}_{p}\right)+W_{\text {nuc }}-W_{\text {coag }}
$$

where $\vec{F}_{p}$ is the particle flux density determined through a drift-diffusion expression as in the case of carbon clusters. $W_{n u c}$ accounts for particle production through nucleation processes and represents therefore the nucleation rate, i.e., the total rate of cluster aggregation processes that lead to more than 30 carbons (30 carbons is the size assumed for the LME). $W_{\text {coag }}$ accounts for the reduction of the particle number density due to aggregation processes that merge two particles in a single one. The source terms are thoroughly discussed in [7]. Note that the image potential enhancement of coagulation rate suggested in reference by Ravi and Girshick is not taken into account in this study [16].

The particle volume mean diameter is estimated from the average mass of particles assuming a material density of 1 g.cm ${ }^{-3}$ [17]. The average particle mass is determined using the following particle mass conservation equation that expresses the balance between the rates of change in particle mass density due to transport fluxes, nucleation and deposition of gas phase species on the surface of solid particulates :

$$
\frac{\partial n_{p} \cdot \bar{m}_{p}}{\partial t}=-\vec{\nabla}\left(\bar{m}_{p} \cdot \vec{F}_{p}\right)+W_{n u c} \cdot m_{n u c}+\sum_{i=1}^{n c l u s t e r} W_{s t}^{i} \cdot m_{\text {cluster }}^{i}
$$

$m_{n u c}$ is the mass of the nucleus and $\mathrm{m}_{\mathrm{i}}$ the mass of the cluster $\mathrm{i}$. The source terms, i.e. last two terms of the right hand side, that appear in this equation account for particle mass gain through nucleation and sticking of carbon clusters on the existing particles. 
The discharge conditions encountered in the NG and the FDS are such that the particle charge may significantly deviate from the steady state charge obtained when assuming that charging process is only due to electron and positively charged ion fluxes. This deviation is due to the contribution of nucleation of singly charged particles and sticking of charged clusters to the particles in the average charge governing equation (3). Note also, that the electron temperature for the dominant cold electrons is very low, i.e., $0.1 \mathrm{eV}$ [18]. Consequently, the charging of particles, especially those with diameter below $10 \mathrm{~nm}$, is characterized by a fairly long characteristic time. A $4 \mathrm{~nm}$ particle needs $0.3 \mathrm{~s}$ in order to reach its equilibrium charge of -0.8 elementary charge whereas a $10 \mathrm{~nm}$ particle will reach -2 elementary charge in a few $\mathrm{ms}$ (cf. figure 1).

As a result, we have to consider the dynamic evolution of the average charge of solid particles through the following charge conservation equation that expresses the balance between the rates of change of particle charge due to transport effects, absorption of electron (slow and fast), positive ion and negative clusters, as well as nucleation through charged cluster.

$$
\frac{\partial n_{p} \cdot \bar{q}_{p}}{\partial t}=-\vec{\nabla}\left(\bar{q}_{p} \cdot \vec{F}_{p}\right)+\left(I_{e}+I_{i}+I_{e}^{h}\right) \cdot S \cdot n_{p}+W_{n u c} \cdot q_{n u c}+\sum_{i=1}^{n c l u s t e r} W_{s t}^{i} \cdot q_{\text {cluster }}^{i}
$$

Where $q_{n u c}$ is the charge of the nucleus, $q_{\text {cluster }}^{i}$ the charge of the cluster $\mathrm{i}, S$ the particle averaged surface and $I_{j}^{k}$ the courant density expressed using OML theory. In this equation we distinguish charging due to fast $I_{e}^{\text {fast }}$ [19], intermediate $I_{e}^{\text {int }}[19]$, and cold $I_{e}^{\text {cold }}$ electrons [20]. Of course charging due to ions is also taken into account $\left(I_{i}\right)$.

Equation (4) allows estimating the average charge of the particles. This is not sufficient since describing particle growth through coagulation and surface sticking of clusters requires in principle the knowledge of the detailed charge and size distributions of the particles. As a matter of fact, the major driver for particle coagulation in dusty plasma is charge fluctuation effect that results in the presence of neutral or positively charged particles, especially for small size particles [21]. Assuming the same average negative charge for all particles would prevent any coagulation. Similarly, particle charge fluctuation enhances surface sticking of negatively charged clusters. It is therefore necessary to take into account the charge fluctuation effect. For this purpose we make use of the approach proposed by Matsoukas et al. [22] who derive an analytic expression for charge distribution as a function of the average size and charge of the particles and of the plasmas parameters, $T_{e}$ and $n_{e}$. Note however, that the validity of the analytic expression given in [22] is questionable for small size particles. Nevertheless, we estimate the fraction of particle that may be neutral or positively charged as a result of charge fluctuation over all the considered size domain. The charge distribution of 5 $\mathrm{nm}$ particles with averaged charge of -1 and -5 obtained for $\mathrm{Te}=0.1 \mathrm{eV}$ are given in figure 2 .

In practice, beside the very dominant negatively charged particles only neutral and singly charged positive particles contribute significantly to coagulation and surface growth processes [23].

The boundary conditions for the particle density continuity equation are similar to those used for the cluster density continuity equation. The boundary conditions for mass and charge density conservation equations are derived on the basis of consistent density, mass and charge fluxes at the anode and cathode surface. This is discussed in detail in Reference [7]. 


\section{II.4. Cold electron transport, and dusty plasma effect in the NG and FSD,}

The dusty plasma effect is basically described through the transport of the cold electron population. As mentioned previously the model developed here considers that the discharge current is carried out by the intermediate energy electrons. This results in approximately zero current carried out by the other charged species. In other words, the present model assumes that the net electrical current carried out by the positive ions, the negative clusters, the negative particles and the dominant cold electron is zero. This is equivalent to assuming that the transport of cold electrons, positive argon-ions and negative clusters and particles is ambipolar.

Consequently, the plasma can be assumed to be locally neutral in the NG and the FDS and the constraint of zero electrical current for the cold electrons and ions enables determining the electric field in these regions. We recall that the discharge current is carried by the intermediate electrons.

Therefore to describe the discharge equilibrium one has to consider a transport equation for slow electrons (or argon-ion), the density of argon-ion (or slow electron) being determined form a local electrical neutrality constraint.

Here we use the following transport equation for cold electrons that expresses the balance between drift-diffusion flux, non local ionisation rate (in the NG), attachment processes, absorption on dust particles and losses at the reactor wall:

$$
\frac{\partial n_{e}}{\partial t}=-\vec{\nabla}\left(-D_{e} \vec{\nabla} n_{e}+\mu_{e} n_{e} \vec{E}\right)+S_{i}(x)-P_{d u s t}^{e}-P_{\text {attach }}^{e}-P_{\text {rad }}^{e}\left(\frac{\sum n_{-}}{n_{e}}\right)
$$

where $D_{e}, \mu_{e}$ are the electron diffusion and mobility coefficients, $S_{i}$ the ionization source term obtained from a Monte-carlo simulation of the fast electron population combined with an analytical sheath model [18] (this term is disregarded in the FDS), $P_{\text {dust }}^{e}$ and $P_{\text {attach }}^{e}$ are the electron losses on the particles and clusters and $P^{e}$ rad is the electron loss on the wall which is controlled by ambipolar diffusion. The ambipolar diffusion coefficient is a function of the neutral carbon species density, $n_{-}$[24].

The structure of the electric field in the NG and FDS regions, and therefore the field reversal effect if it takes place, are determined by expressing that the electrical current carried out by the cold electron, the positive ion and the negative clusters and particles is zero:

$$
E=\frac{D_{+} \nabla n_{+}-D_{e} \nabla n_{e}-\sum Z_{-} D_{-} \nabla n_{-}}{\mu_{e} n_{e}+\mu_{+} n_{+}+\sum Z_{-}^{2} \mu_{-} n_{-}}
$$

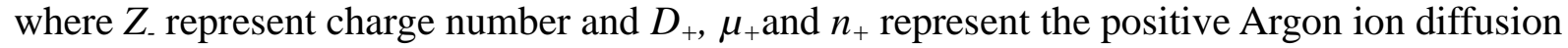
coefficient, mobility coefficient and density.

It is worthy to mention hear that the approach used here is similar, at least in its spirit, to that proposed by Boeuf and Pitchford [25]. The main difference comes from the fact that we take into account the radial losses of charged species on the lateral wall of the reactor. This approach is quite different from the one that is proposed by Kolobov and Tsendin [18] and that we used in our previous work on the early discharge phase where the plasma is still electropositive and the clusters and particles do not contribute to the discharge equilibrium. In this approach, the E-field is derived assuming that the cold electrons satisfy the Boltzmann equilibrium. Note that for both these approaches cold electron temperature is one of the keyparameter of the model that is very difficult to estimate. As discussed by Kolobov and 
Tsendin, measurement tends to indicate that the temperature of these electrons would be of few tenths of eV [26]. The authors investigated the effect of these parameters on particle formation and showed that the particle formation can only take place in a narrow window of cold electron temperature that is consistent with the value reported in [18] and that would be approximately $0.1 \mathrm{eV}$ [7].

Basically, equation (5) and (6),along with the conservation equations for cluster densities (1), and solid particle, number (2), mass (3) and charge (4) lead to a self consistent description of the E-field and charged species densities distributions in the NG and the FDS, which makes it possible to describe the dusty plasma effect in these regions.

\section{II.5. Solution method}

The model discussed in the previous sections end up with a description of the time-space variations of cluster density, dust particle density, dust particle average mass, dust particle average charge, and electron density through a set of 130 non linear partial differential equations that are strongly coupled and subject to a non linear differential constraint that yield the electric field.

The simulation domain is located between the sheath edge and the anode considering only a short discharge. This means that the sheath region is disregarded when investigating the dusty plasma effect. This should not affect the results since negative cluster and particles are produced outside the sheath, in the NG and the FDS. As for the boundary conditions, we assume zero density for cold electron and negative clusters and particles at the sheath edge. The boundary conditions at the anode for negative clusters and solid particles express the balance between the rates of transport flux and sticking processes. For cold electrons, we assume a zero density at the end of cathode sheath and zero gradient condition at the anode, this means that the cold electron flux are absorbed at the anode, their flux being essentially a drift flux.

As for the numerical approach, we used a central difference approximation for diffusiondominated fluxes and an upwind approximation for (drift) convection-dominated flux. This formulation transforms the partial differential equation system into a large system of coupled non-linear ordinary differential equations that are solved by time integration using the Backward Difference Formula (BDF) method.

However a full implicit treatment of such a coupled system of equations is quite difficult due to an ill-conditioned jacobian matrix that results at least partly from the strong coupling between the transport fluxes of carbonaceous species and the density of cold electrons which determine the ambipolar electric field dynamics. Therefore, since the characteristic time for the density variation of clusters, of the order of few seconds $\left(\tau_{c l 5} \sim 10 \mathrm{~s}\right)$, is much greater than the cold electron plasma frequency $\tau_{e} \sim 10^{-5} \mathrm{~s}$, we used an explicit treatment for the estimation of the carbonaceous transport fluxes as function of the electric field. The flux at a time $t+d t$ is estimated as a function of the field at $t$ using:

$$
\vec{F}^{t+d t}{ }_{i}=-D_{i} \vec{\nabla} n_{i}^{t+d t}+\vec{z}_{i}^{t+d t} \mu_{i} n_{i}^{t+d t} \overrightarrow{E^{t}}
$$

The change in cold electron density shows a much shorter characteristic time and an implicit formulation was used. An under-relaxation is introduced to enable a system of differential equation with a diagonally dominant Jacobian matrix.

$\vec{F}_{e}^{k+1}=-D_{e} \vec{\nabla} n_{e}^{k+1}+\bar{z} \mu n_{e}^{k+1}\left((1-\varepsilon) \vec{E}^{k+1}+\varepsilon \vec{E}^{k}\right)$

where $\varepsilon=0,1$. 


\section{Results}

\section{III.1. Simulation conditions}

In the simulations described in paper, the cold electron temperature is set to $0.1 \mathrm{eV}$.

The carbon cluster populations are followed until they reach 30 carbons with different isomer configuration. We consider that these carbon clusters are in thermal equilibrium at $300 \mathrm{~K}$. We assume that the sticking coefficient of the neutral clusters on particle surface is 1 . Finally 126 continuity equations are considered.

\section{III.2. Dynamic of the discharge at the field reversal position}

Figure 3 shows the axial profiles of the electron density and the axial electric field between the cathode, $x=0 \mathrm{~cm}$, and the anode, $x=14 \mathrm{~cm}$. The electron density, Figure 3.a, reaches a maximum of $10^{11} \mathrm{~cm}^{-3}$ in the negative glow, at a distance of $2.25 \mathrm{~cm}$ from the cathode. The Efield profile, Figure 3.b, shows a reversal at a $2.3 \mathrm{~cm}$ in the negative glow. Since the discharge is short, i.e., no positive column, the field remains positive in the whole region between the reversal position and the anode. The field remains almost constant, at a value around $5 \times 10^{-2}$ $\mathrm{V} . \mathrm{cm}^{-1}$, in a large fraction of this region. Then, it decreases in the vicinity of the anode. Note that the field reversal is weaker by more than 8 orders of magnitudes than the field in the cathode fall, i.e., $5 \times 10^{3} \mathrm{~V} . \mathrm{cm}^{-3}$.

Figure 4a shows the time evolution of the negatively charged $\mathrm{C}_{4}{ }^{-}, \mathrm{C}_{15}{ }^{-}$et $\mathrm{C}_{30}{ }^{-}$clusters at the position of the field reversal, i.e., $\mathrm{E}=0$. The density of the clusters increases very sharply during the first 150-200 s of the discharge. The maximum density of the $\mathrm{C}_{4}{ }_{4}^{-}$is around $10^{9} \mathrm{~cm}^{-}$ 3 , while the density of $\mathrm{C}_{30}{ }^{-}$that is responsible for solid particle nucleation remains below $10^{7} \mathrm{~cm}^{-3}$. The time-variations of the neutral cluster densities are shown Figure 4b. The densities of neutral clusters with more than four carbon-atoms, are much smaller than the corresponding negative cluster densities. The neutral clusters are indeed rapidly lost by diffusion to the wall, while the negative clusters are trapped in the field reversal. The only neutral species that show large densities are $\mathrm{C}, \mathrm{C}_{2}$ and $\mathrm{C}_{3}$ that are sputtered from the cathode (cf. figure 5). Actually, aggregation processes between the high density small neutral clusters that are emitted at the cathode result in the formation of $\mathrm{C}_{4}-\mathrm{C}_{6}$ neutral clusters, a fraction of which undergoes attachment processes that result in the production of $\mathrm{C}_{4}^{-}, \mathrm{C}_{5}^{-}$and $\mathrm{C}_{6}^{-}$ negative clusters. These small negative clusters are trapped in the field reversal region where they undergo a molecular growth process that end up with the nucleation of solid particles. This molecular growth route is schematized in figure 6 .

Figure 7 shows the time-variation of the solid particle density (figure 7a), diameter (figure 7b) and charge (figure $7 \mathrm{c}$ ) at the field reversal position, i.e., $\mathrm{E}=0$ position. The particle density increases during the 150-200 s, reaches a value of $2 \times 10^{9} \mathrm{~cm}^{-3}$, slightly decreases for timeperiods between 300 and $500 \mathrm{sec}$ and then remains almost constant for longer durations. The particle diameter shows a different behaviour since it increases steadily, reaching $30 \mathrm{~nm}$ at $200 \mathrm{~s}$ and $60 \mathrm{~nm}$ at $600 \mathrm{~s}$. The particle charge number increases steadily up to 6 negative charges for a particle diameter of $60 \mathrm{~nm}$ (The net charge, which is the opposite of the charge number decreases).

To understand the time-variation of particle density and average diameter, we report in figure 8 the contributions of the different phenomena involved in the particle formation and growth. Figure 8a shows that the nucleation rate, the number of solid particles produced per unit time and volume, remains greater than the number of solid particles consumed by coagulation. The ratio of the nucleation to the coagulation rates is around 100 for short time-period, i.e., when the particles start to form, and decreases significantly at longer time period to less than a factor 10 when the particle density becomes significant, i.e. around $10^{9} \mathrm{~cm}^{-3}$. It appears 
therefore that the nucleation rate always exceeds the coagulation rate during the whole discharge duration. This means that there is a continuous net production of particles at the field reversal position. Nevertheless, particle density at this position reaches a maximum of $3 \times 10^{9} \mathrm{~cm}^{-3}$ at a time-value of $300 \mathrm{~s}$ and slightly changes above this value. The limitation in the particle density despite a positive net production of particles is due to transport effects and changes in the topology of the electric field in the vicinity of the field reversal position. Indeed and as will be discussed in the next section, the particles that are produced at the field reversal position are transported by drift-diffusion in the surrounding region. The change in the field topology and the resulting transport processes even results in a slight decrease of the particle density at the field reversal position for time-periods between $300 \mathrm{~s}$ and $500 \mathrm{~s}$ despite the positive net production rate. Figure $8 \mathrm{~b}$ shows a comparison between the contributions to particle growth, i.e., the increase of the mean diameter, due to the sticking of carbon clusters on one hand, and to the particle coagulation on the other hand. These contributions are expressed in terms of the number of carbon atoms gained by each solid carbon-particle per unit time. Basically, the particle growth is mainly insured by the sticking of carbon cluster. The number of carbon atoms gained by each particle through this process remains at least two-orders of magnitude higher than the one corresponding to the coagulation process. Figure $8 \mathrm{c}$ shows the contribution of the different clusters to the particle growth through molecular deposition at the surface. It appears clearly that the small clusters emitted at the cathode bring the major contribution to particle growth and therefore to the observed increase of the particle diameter. This result may be well understood when considering that the major part of the large clusters, i.e., $\mathrm{C}_{\mathrm{n}>3}$, are negatively charged. Their sticking to the negatively charged particle is therefore strongly inhibited by the electrostatic repulsive forces. Note that the 2 orders of magnitude decrease in the nucleation rate over the time-periods ranging between 200 and 600 $\mathrm{s}$ is consistent with the one order of magnitude decreases of the $\mathrm{C}, \mathrm{C}_{2}$ and $\mathrm{C}_{3}$ densities on one hand and of the $\mathrm{C}_{4}{ }^{-}$density on the other hand. The rate of molecular growth that determine the nucleation kinetics is indeed at least partly determined by the second order aggregation processes between the negative clusters, like $\mathrm{C}_{4}^{-}$, and the dominant small neutral clusters emitted at the cathode.

Figure 9a represents the time variation of the electron density at the field reversal position. This density remains almost constant during the first $150 \mathrm{~s}$, it decreases exponentially by more than one order of magnitude for time-periods ranging between 150 and $300 \mathrm{~s}$, and at a much slower rate for longer time-periods. The values obtained for the electron density at the field reversal position and for time period of 500-600 s are in quite good agreement with those measured by Dominique et al. [27] who found a value of approximately $10^{10} \mathrm{~cm}^{-3}$ at $2.5 \mathrm{~cm}$ from the cathode.

The decrease of the electron density is accompanied by a strong increase of the negative clusters and charged particle densities. The total electrical charge carried by negative clusters and charged particles exceeds the electrical charge carried by electrons as shown in figure $9 \mathrm{~b}$ that depicts the discharge electronegativity factor $\alpha$. The discharge becomes therefore electronegative at the field reversal position. The analysis of the loss terms, depicted in figure 10 , shows that the electron depletion is mainly due to the losses at the reactor wall during the first 100 seconds of the discharge and to the dust particle charging for longer discharge duration. It is also interesting to notice that the charging of the dust particles becomes rapidly the major electron loss process despite the very small value of the cold electron temperature, i.e., $\mathrm{T}_{\mathrm{e}}=0.1 \mathrm{eV}$, that results in very limited electron attachment charging rates as compared to what is usually obtained in capacitively coupled RF discharges, for $\mathrm{T}_{\mathrm{e}} \sim 2 \mathrm{eV}$. 


\section{III.3. Space distributions of the charged species densities, self consistent E-field and dust cloud densities, average charge and mean diameter.}

So far, we mainly discussed the time-evolution of the discharge parameters at the field reversal position. Cluster and particle formation and the resulting time-evolution of the discharge parameters significantly depend on the considered discharge region. We therefore discuss in the second part of this section the evolution of the axial distribution of the different discharge parameters. Figure 11 shows the axial profile of $\mathrm{C}_{4}{ }^{-}$density for time periods of 100 , $150,200,400$ and $600 \mathrm{~s}$. The cluster density is significant, i.e., density-values greater than $1 \%$ of the maximum value, in a region located between 1 and $6 \mathrm{~cm}$ distance from the cathode. Although the maximum value decreases significantly for time period above 150-200 s, the region with large density value tends to extend significantly, and the maximum density-value is reached over a $2-3 \mathrm{~cm}$ plateau with a constant density. The axial profiles of larger cluster densities show similar relative variations, although the absolute values are one and two orders of magnitude smaller for $\mathrm{C}_{15}{ }^{-}$and $\mathrm{C}_{30}$, respectively. Also, axial profiles with a plateau region are obtained earlier for larger clusters. They are observed at a time period of $400 \mathrm{~s}$ for $\mathrm{C}_{30}{ }^{-}$and $600 \mathrm{~s}$ for $\mathrm{C}_{4}{ }^{-}$.. Similar behaviour are obtained for the electron density, since, as shown in Fig. 12, the large decrease of the maximum electron density, i.e., more than one order of magnitude, is accompanied with the formation of a large plateau region where the electron density reaches its maximum value. The transition from an electropositive to an electronegative discharge is accompanied with a strong decrease of the ionization degree. This decrease in the total ionization degree may be well understood when considering that electron and positive ion production through the non local ionization is very weakly affected by the formation of carbon clusters and particles and remains therefore almost constant meanwhile electron and positive ion losses strongly increase with the appearance of solid particles (cf. figure 10).

The dust density profiles calculated for 100, 150, 200, 400 and $600 \mathrm{~s}$ are shown in figure 13. Basically, during the early time period, the dust cloud shows a density profile with a pronounced maximum. This maximum is reached at the field reversal position at the early stage of the discharge $(200 \mathrm{~s})$ and tends to move toward the sheath for larger time periods (400 s). The maximum particle density shows only a slight decrease for time-periods between 400 and $600 \mathrm{~s}$, where the major change observed is the spreading of the dust cloud and the formation of a plateau region where the maximum density is reached (see the dust density distribution at $600 \mathrm{~s}$ ). Basically, the evolution of the dust cloud formed in the investigated discharge is characterized by a first phase where the cloud shows a significant non-uniformity in terms of density, and a second phase where the dust cloud tends to spread out with a large region of maximum concentration (a plateau shape) that remains almost constant and around $1-2 \times 10^{9} \mathrm{~cm}^{-3}$. This is somewhat larger than the estimate derived experimentally by laser extinction [10] who suggested a value of $2 \times 10^{8} \mathrm{~cm}^{-3}$ for a time-period of $600 \mathrm{~s}$. This difference may be due to the absence of any detailed representation of the particle size distribution and the subsequent approximate treatment of the coagulation kinetics. The model describes the particle clouds only in terms of averaged quantities, i.e., number density, diameter and charge, and is therefore quite simple as far as aerosol dynamics is concerned. Nevertheless, the model prediction and the experimental estimation of the particle number density remain of the same order of magnitude which can be considered as satisfactory.

Dust particles are therefore trapped in a region located between the sheath edge and the bulk of the discharge, namely the negative glow of the DC discharge. This is similar to what was 
already observed in several experiments on similar or different discharge systems [28]. However, the position and width of the dust cloud highly depend on the space-distribution of the E-field shown in figure 14. As a matter of fact, a constant electric field region, consistent with the plateau observed in the charged species density profiles, is also observed for large time periods, i.e., above $400 \mathrm{~s}$ (figure 14). The formation of this constant E-field region is accompanied with a decrease of the electric field magnitude (in absolute value) around the initial field reversal position. This evolution ends up with an almost zero E-field magnitude over a wide region between the initial field reversal position and the sheath. Compared with an electropositive discharge, the field structure in the dusty electronegative discharge does not show a precise field reversal position, we rather obtain a negative E-field in the region of the negative glow that is close to the sheath, a wide almost zero E-field region in the bulk of the negative glow and a positive E-field in the negative glow and the Faraday dark space. This zero-field quasi-equipotential region corresponds to space occupied by the dust cloud. Outside the dust cloud in the NG and FDS regions the electric field is almost not affected by the presence of the particles and its magnitude remains nearly equal to the one obtained in the pristine argon discharge.

Figure 15 shows the space distribution of the particle mean-diameter (15. a) and average charge (15.b). Of course, the particle mean diameter increases with time and the maximum mean-diameter values varies from $30 \mathrm{~nm}$ at $200 \mathrm{~s}$ to $50 \mathrm{~nm}$ at $400 \mathrm{~s}$ and $75 \mathrm{~nm}$ at $600 \mathrm{~s}$. This increase is in good agreement with experimental measurements of Dominique et al [5], showing that mean sizes of $\sim 35 \mathrm{~nm}$ and $\sim 45 \mathrm{~nm}$ are reached at $180 \mathrm{~s}$ and $420 \mathrm{~s}$, respectively. Note however that mean sizes determined experimentally are number mean diameter and may therefore differ from the calculated volume mean diameter. Basically, a volume mean diameter is larger than the corresponding number-mean diameter. This would indicate that the calculated sizes are slightly smaller than the measured one.

Nevertheless, this result can be considered as a very satisfactory since the model used does not describe the details of the particle size distributions an only considers average quantities to characterize the particle cloud. The present model can be therefore used to investigate, at least qualitatively and more probably semi-quantitatively in terms of variations and trends, the major effects that govern the dusty plasmas obtained in DC sputtering discharges.

The space distribution of the dust mean-diameter becomes strongly non uniform for larger time-periods. As a matter of fact, while the space distribution for short discharge periods, i.e., $\mathrm{t}=200 \mathrm{~s}$, shows a maximum value at the field reversal position, in the bulk of the dust cloud, and almost symmetric behaviour with smaller diameter around the field reversal position, the distribution at large time periods tends to becomes much less symmetric with a maximum diameter reached at the edge of the dust cloud facing the cathode sheath and a strong decrease of the mean diameter with the distance from the cathode. In other words, the largest dust particles tend to accumulate at the edge of the cloud facing the cathode sheath while the particles inside the cloud are smaller. This result may be well understood by comparing the different source terms involved in the particle growth kinetics. As a matter of fact, particle growth takes place through surface sticking of the small clusters sputtered from the cathode. These clusters interact first with the particles located in the cloud region facing the cathode. Their density significantly decreases inside the dust cloud as shown in figure 16 in the case of $\mathrm{C}$-atom. Therefore, dust particle growth through carbon cluster sticking is much less effective for larger distance from the cathode. As a result, while the axial distributions of the $\mathrm{C}_{2} \mathrm{C}_{2}$ and $\mathrm{C}_{3}$ cluster densities exhibit a simple exponential behaviour at short time periods, i.e., $\mathrm{t}<200 \mathrm{~s}$, they evolve to bimodal distributions for larger time periods, i.e., $\mathrm{t}=400$ and $600 \mathrm{~s}$ (cf. figure 16 for the case of C-atom density). This bimodal distributions show a first exponential part with a strong variation, i.e., more than one order of magnitude over $2 \mathrm{~cm}$ distance. This happens in a region located between the cathode and the left side of the dust cloud where the particles 
have the largest mean-diameter and induce an enhanced loss of the small neutral carbon clusters through heterogeneous surface reaction $(0<x<2.5 \mathrm{~cm})$. In this first region, the variation rate of the carbon cluster density with the distance from the cathode strongly increases with the time period. It is therefore consistent with the trends of the variation of the maximum value of the particle mean diameter obtained at the cathode faced side of the particle cloud with the time-period. The second part of the distributions corresponds to distances above $3 \mathrm{~cm}$ from the cathode, and shows much slower and similar values for the variation rates of the carbon cluster density as function of the distance over the whole timedomain explored in this work (cf. Figure 16 for the case of C-atom density).

Further understanding of the interplay between the different phenomena that govern the particle production and growth kinetics may be gained by comparing the source terms involved in the particle number and mass density equations. For this purpose, we reported the axial profiles of the coagulation and nucleation rates for different time-periods in figure 17. This shows that the maximum nucleation rate decreases by one order of magnitude between 200 and $600 \mathrm{~s}$ (figure 17.a). The position of the maximum is however shifted toward the sheath at the edge of the dust cloud. The decrease of the nucleation rate with time inside the cloud is much more pronounced since the rate varies by two orders of magnitude between 200 and $600 \mathrm{~s}$. This means that although the nucleation still takes place at the edge of the dust cloud facing the sheath, it is clearly quenched in the bulk of the cloud and in the whole region located between the cloud and the anode. Figure 17.b shows the axial profiles of the coagulation rate for the same time periods. The coagulation rate strongly decreases in the region where largest mean-diameters are obtained, i.e., the cathode-faced side of the dust cloud facing the sheath. The decrease of the coagulation in the bulk and in the right side of the dust cloud remains however quite moderate, i.e., less than one order of magnitude, and the coagulation rate almost compensates the nucleation rate in the bulk of the cloud, i.e., $x=2-2.5$ $\mathrm{cm}$, and exceeds it in the right side of the cloud, i.e., $\mathrm{x}=2.5-4 \mathrm{~cm}$ for long time periods.

In figure 18 we reported the axial profiles of the carbon gain per particle and per second corresponding to the coagulation processes (Figure 18. a) and to the surface deposition processes (figure $18 \mathrm{~b}$ ). We can see that the rate of a particle growth due to the deposition of carbon clusters on its surface exceeds by 1-4 orders of magnitude depending on the considered position. The evolution of the particle mean diameter is then clearly largely dominated by surface sticking of carbon clusters.

The analysis above enables to draw a simple picture for the aerosol dynamics that characterize the dusty plasma obtained in sputtering DC discharge. The particle number density is mainly governed by the balance between (i) nucleation processes, the only particle production process, at the edge of the dust cloud facing the sheath potential barrier, where the small carbon clusters emitted at the cathode still shows high density-values to initiate molecular growth, (ii) coagulation processes, the only particle number reduction process, in the bulk and in the anode-faced side of the cloud where particle mean diameter and average charge are small enough to make the formation of neutral or positively charged particles through charge fluctuation significant enough thus enhancing coagulation and (iii) transport phenomena that insure the transfer of a fraction of the particles that are produced in the nucleation region to the coagulation region (without affecting the global number of particles), thus leading to almost constant solid particle density in the major fraction of the dust cloud. The particle size is mainly governed by the deposition of small neutral carbon clusters on particle surface. The coagulation processes, as modelled in this work, do not affect particle size significantly, while they play a key-role in affecting the particle number density in the bulk of the cloud and in the cloud region facing the anode.

The axial distributions of the average charge of the dust particles obtained for different time periods (cf. Figure 15.b) show behaviours that are similar to those of the mean diameter 
distributions. Basically, for long time-periods, particles with the largest charge (in absolute value), i.e., around 7-8 charge units, corresponds to those with the largest diameter and are therefore located at the edge of the dust cloud facing the cathode sheath. Consistently, the smaller particles located inside the dust cloud and farther from the cathode sheath shows smaller charges (in absolute value), i.e., 2-4 charge unit. Also interesting is the comparison of the calculated average-charge and the charge value that would be obtained using OML theory. These are also reported figure $15 \mathrm{~b}$ (open symbols). There is a significant deviation of the calculated non-equilibrium average charge from the equilibrium OML charge. For the short time periods, i.e., $t=200 \mathrm{~s}$, the deviation from the OML theory is around 1 charge unit at the edge of the dust cloud and 2 charge units in the bulk of the charge cloud, which corresponds to around $50 \%$ deviation for both cases. For very long time periods, i.e., $t=400-600 \mathrm{~s}$, the deviation from OML is mainly significant at the cloud edge facing the cathode sheath where the difference with respect to OML theory is of almost 2 charge units, which represents $25 \%$ $30 \%$ relative deviation. It appears therefore very important to take into account the deviation of the average charge from the OML theory.

\section{Conclusion}

We developed a self-consistent model of particle formation and growth in DC discharge. Discharge equilibrium, molecular transport and growth, and particle nucleation transport and growth are described self-consistently. The model makes use of a detailed description of the nucleation kinetics that results from the molecular growth of carbon clusters, while the aerosol dynamics was described in a simpler and approximate fashion by expressing balance equations on averaged quantities, i. e., number density, average mass and average charge.

The model results in a mathematical description in terms of a large partial differential continuity equations system subject to an algebraic constraint that yields the ambipolar electric field. This system was solved using a high order implicit approach, which makes it possible to simulate the non-stationary evolution of the dusty plasma over large discharge durations despite the very high stiffness of the system.

The simulations showed that the particle formation takes place and a dusty plasma effect is obtained due to the reversal of the axial electric field that enables trapping negatively charged carbon clusters which makes it possible to induce a molecular growth and a nucleation process. Negative particle formation takes place due to attachment processes on the $\mathrm{C}_{4}, \mathrm{C}_{5}$ and $\mathrm{C}_{6}$ clusters obtained through coagulation of the small neutral clusters emitted at the cathode. They also showed that the field topology is strongly affected by the presence of the dust cloud. Instead of a single field reversal position that characterizes electropositive discharges, the dusty plasmas showed a large zero-field region corresponding to the dust cloud. The trapping effect remains however effective and particle formation is still possible. The formation of a dusty plasma is accompanied with the transition from an electropositive to an electronegative discharge with an electronegativity factor of 2 . The cold electron density is mainly depleted due to the charging of solid particles, attachment processes on carbon clusters being quite negligible in the electron balance. We showed that the dust cloud obtained at a long time-period is characterized by an almost constant particle density. The space distribution of the particle size is however much more non uniform, the largest particle being located at the edge of the dust cloud facing the cathode sheath. We explain this result by analyzing the different effect involved in the aerosol dynamics. We showed that the particle density is governed by nucleation at the edge of the dust cloud facing the sheath, coagulation in the bulk and in the anode-faced side of the cloud, and transport inside the cloud, while 
particle size is mainly governed by the deposition of the small neural clusters emitted at the cathode on the particle surface. Comparison of the results predicted by the model discussed here and estimates determined experimentally show that the present model gives a very good prediction of the electron density in the NG and the particle mean diameter. However, the model predictions overestimate the particle number density estimated experimentally although the calculated and experimental values of this parameter remain in a fairly good qualitative agreement. In any case the fairly good qualitative agreement obtained between the model results discussed in this paper and the experimental estimates of reference [27] tends to support the whole picture inferred from the analysis of the model results as far as the interplay between the different phenomena governing the investigated dusty plasmas, i.e., molecular growth, aerosol dynamics, transport, discharge equilibrium, is concerned.

Of course, this model that deals with the description of quite complex plasmas can be substantially improved. In particular, an improved description of the aerosol dynamics by taking into account a detailed description of the particle size distribution is needed. This would enable a more accurate description of the coagulation kinetics and its impact on the behaviour of the whole dusty plasma. Such improvement would hopefully yield a better agreement with experiments as far as particle number density is concerned, which would make the model much more predictive. Also, the approach used here in the case of graphite cathode and carbon (nano)-particles formation can be used for many other dusty plasmas generated in sputtering discharges provided basic data for sputtering yields and molecular growth kinetics are known.

\section{Acknowledgements}

This work is supported in part by contract ANR-09-BLAN-0070-01 of the French National Research Agency, and undertaken under the auspices of the French Research Federation on Controlled Magnetic Fusion (FRFCM).

\section{Figures and figure caption}

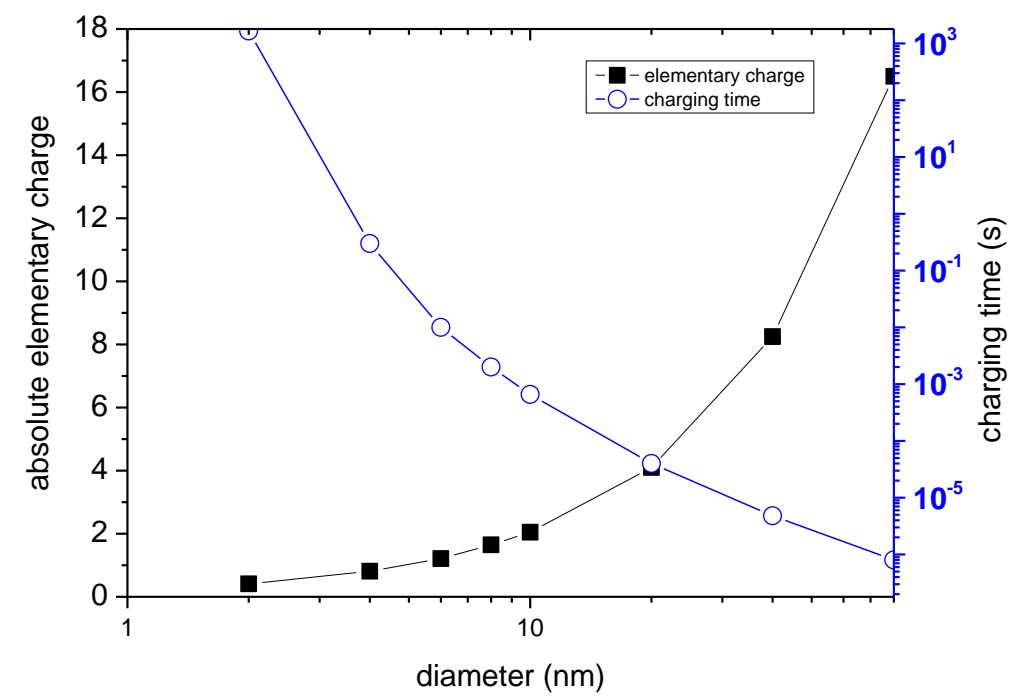


Figure 1 : Absolute elementary charge and charging time derived from the OML theory as functions of the particle diameter for $\mathrm{Te}=0.1 \mathrm{eV}, \mathrm{n}_{\mathrm{i}}=\mathrm{n}_{\mathrm{e}}=10^{10} \mathrm{~cm}^{-3}, \mathrm{Tg}=300 \mathrm{~K}$,

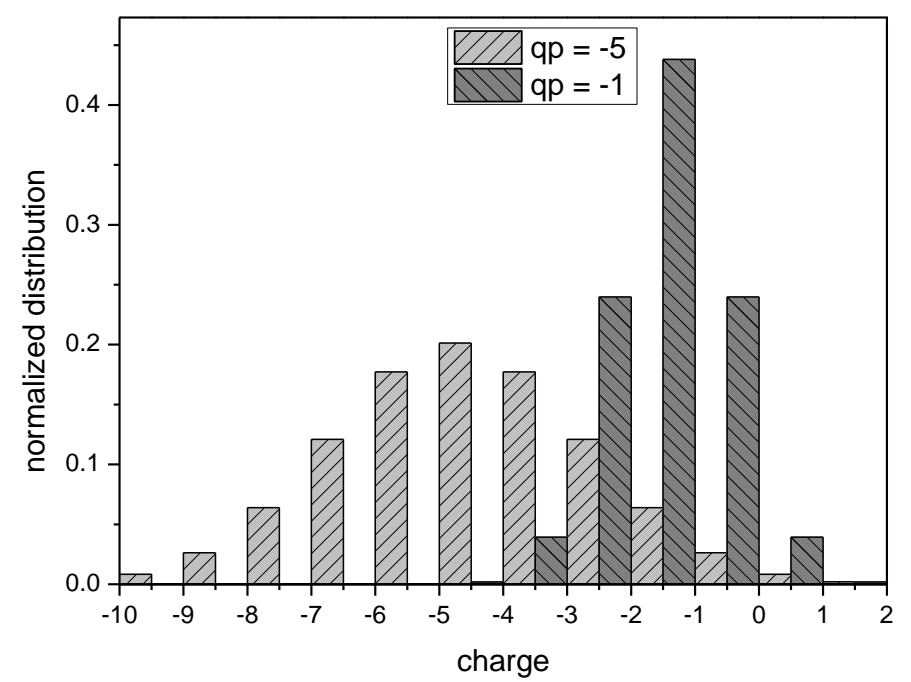

Figure 2 : charge distribution for a $5 \mathrm{~nm}$ particle and an average charge of -5 and -1 for $\mathrm{Te}=0.1 \mathrm{eV}$, $\mathrm{n}_{\mathrm{e}}=\mathrm{n}_{\mathrm{i}}=10^{10} \mathrm{~cm}^{-3}, \mathrm{~T}_{\mathrm{i}}=\mathrm{T}_{\mathrm{g}}=300 \mathrm{~K}$

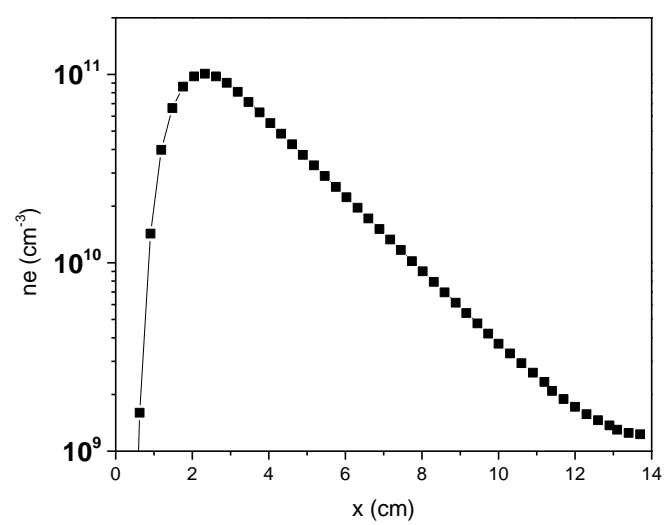

(a)

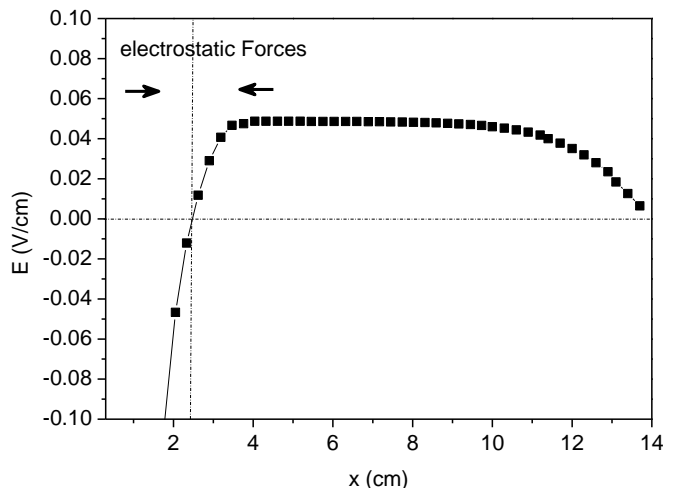

(b)

Figure 3: Axial distribution of the cold electron density (a) and the electric field (b) in the gap region for the pristine argon discharge. The cathode is located at $0 \mathrm{~cm}$ while the anode is at $14 \mathrm{~cm}$. These profiles represent the initial distribution, the distribution at $\mathrm{t}=\mathbf{0} \mathrm{sec}$, for the dusty plasma model. Since the discharge is a short discharge, there is no positive column and the field remains positive beyond the field reversal position, i.e., $x=2.3 \mathrm{~cm}$. 


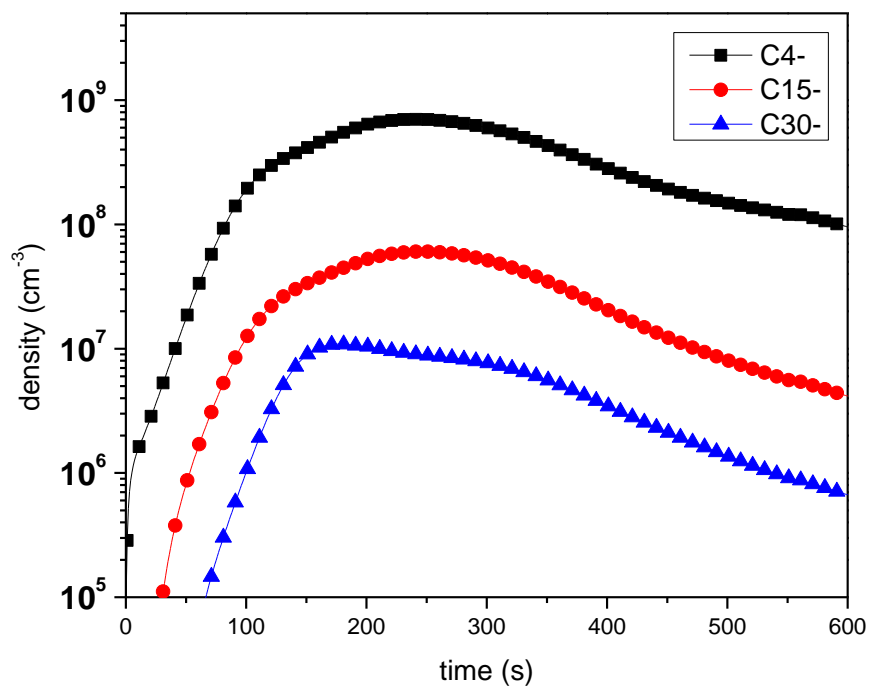

(a)

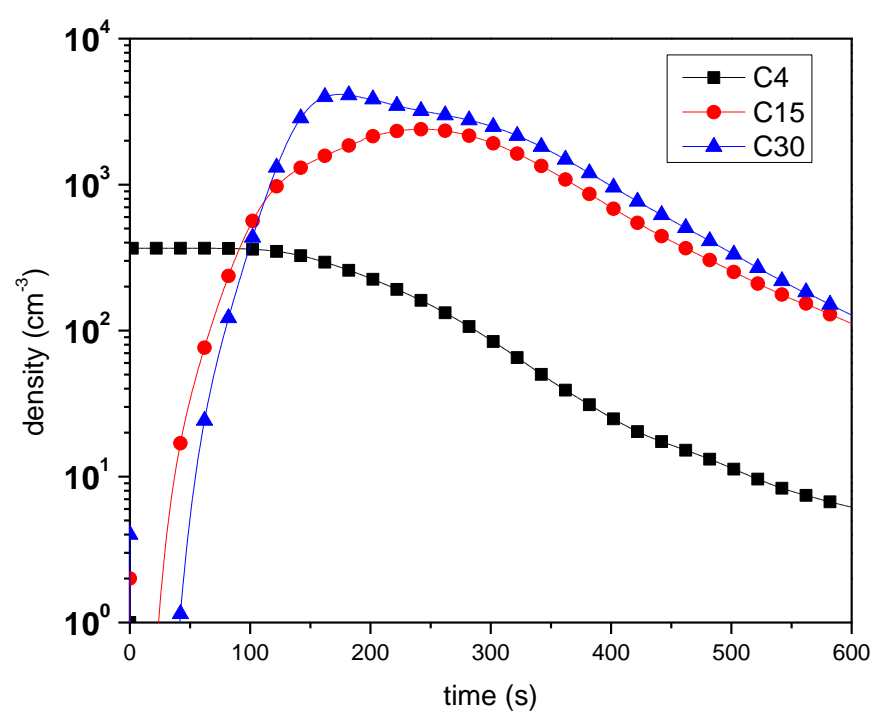

(b)

Figure 4 : Time evolutions of the $\mathrm{C}_{4}^{-}, \mathrm{C}_{15}{ }^{-}$and $\mathrm{C}_{30}{ }^{-}$negative cluster densities (a) and $\mathrm{C}_{4}, \mathrm{C}_{15}$ and $\mathrm{C}_{30}$ neutral cluster densities $(b)$ at the field reversal position, i.e., $x=2.3 \mathrm{~cm}$. 


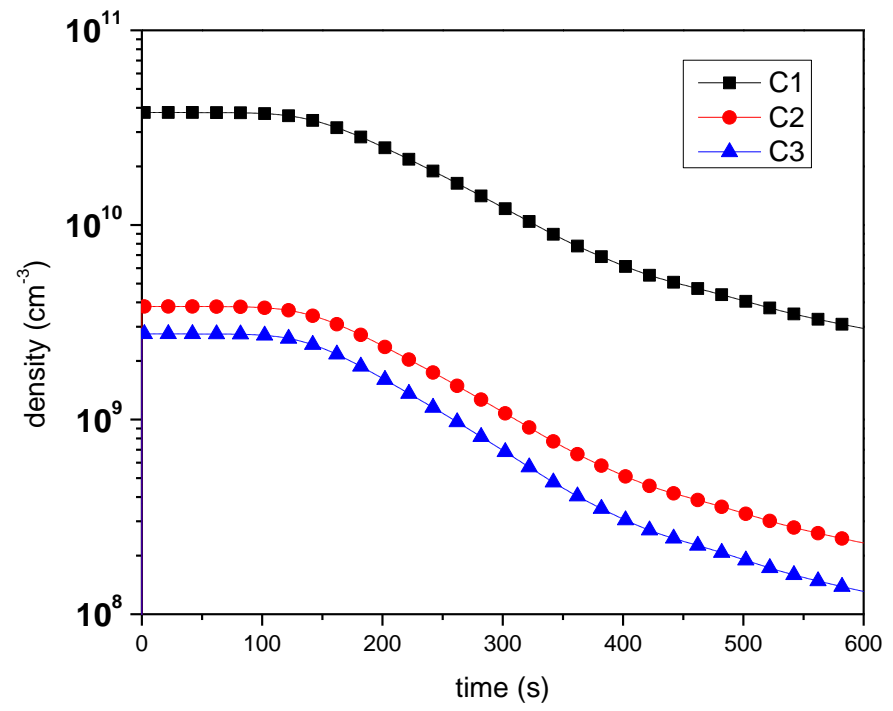

Figure 5 : Time evolutions of the $C, C_{2}$ and $C_{3}$ neutral cluster densities at the field reversal position, i.e., $\mathrm{x}=2.3 \mathrm{~cm}$.

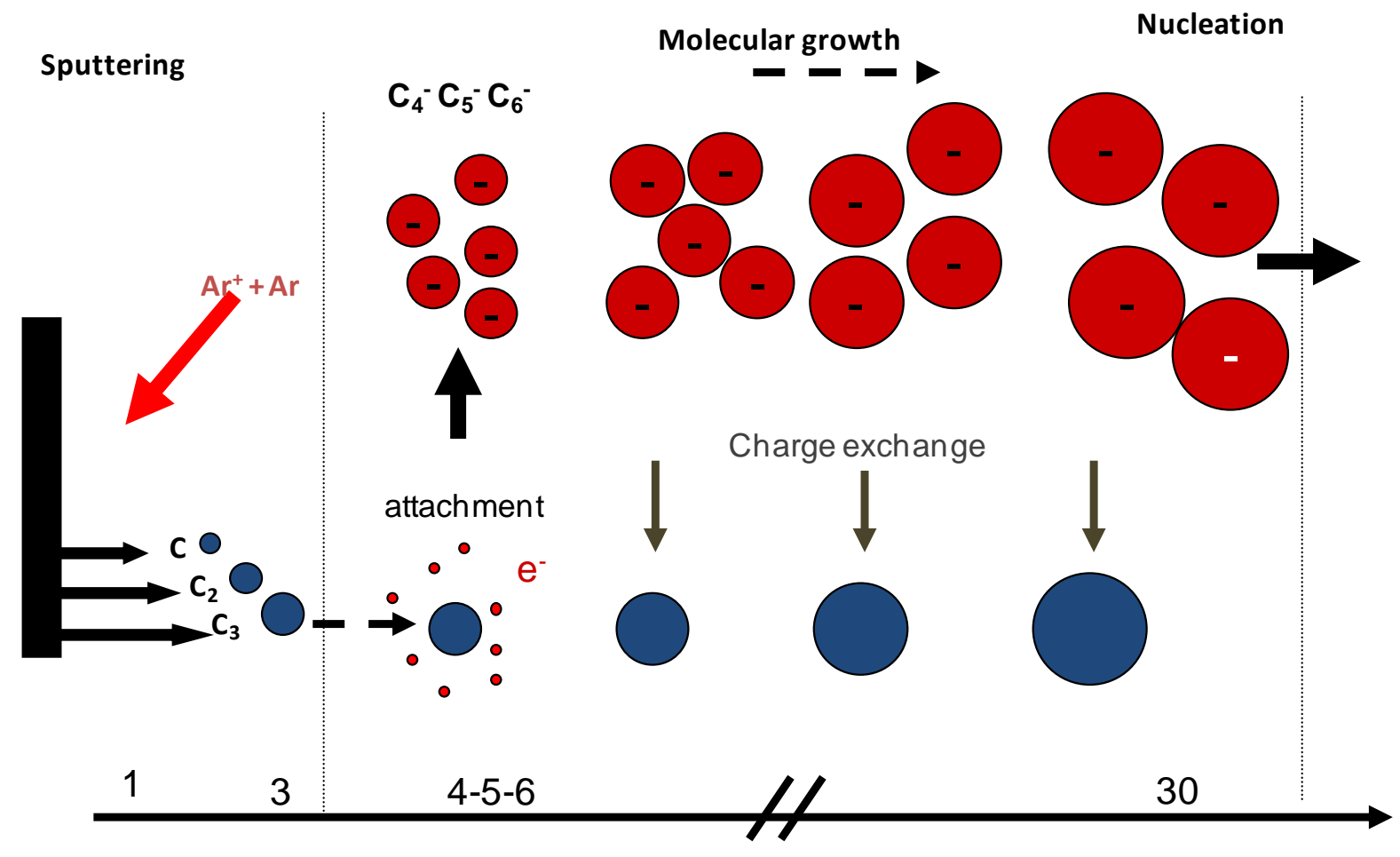

Carbon

Figure 6 : Schematic of the cluster growth route that take place in graphite cathode argon DC discharge and result in the formation of solid particles. 


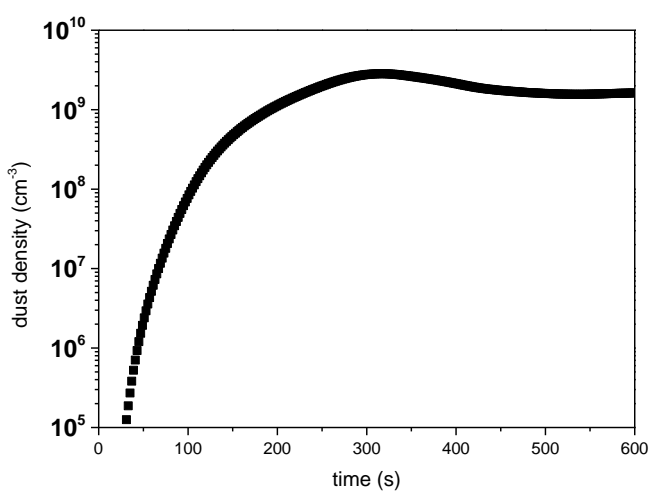

(a)

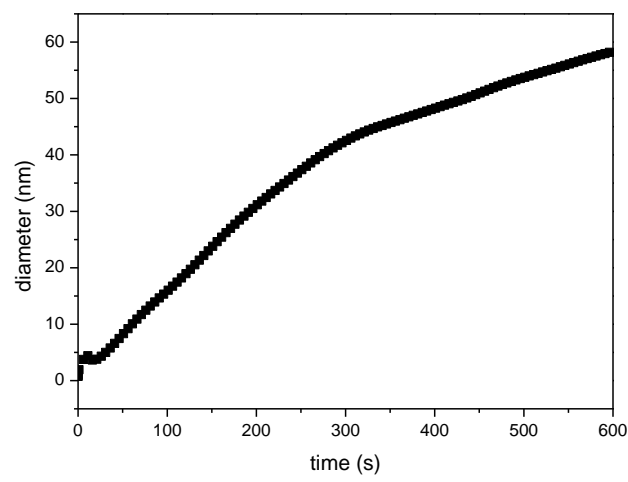

(b)

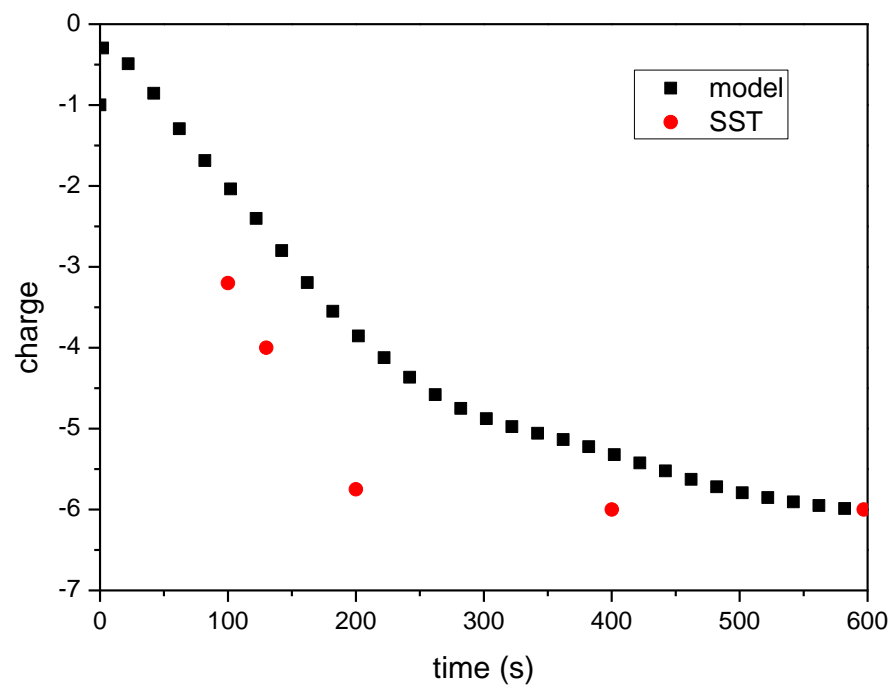

(c)

Figure 7 : Time evolution of the solid particle density (a,) mean-diameter(b)and mean-charge (c) at the field reversal position, i.e., $x=2.3 \mathrm{~cm}$. The average charge obtained at steady state and disregarding the contribution of nucleation of singly charged particles and sticking of negatively charged clusters is also shown and indicated by (SST)

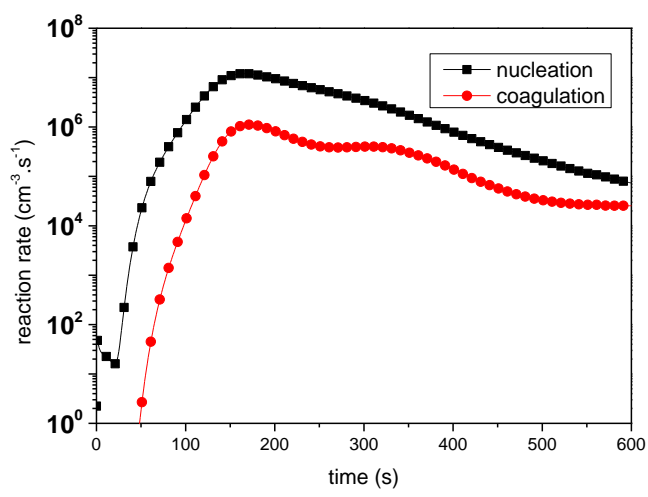

(a)

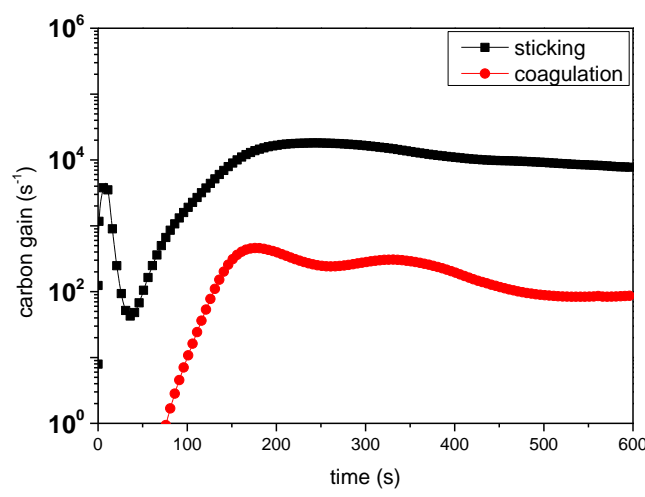

(b) 


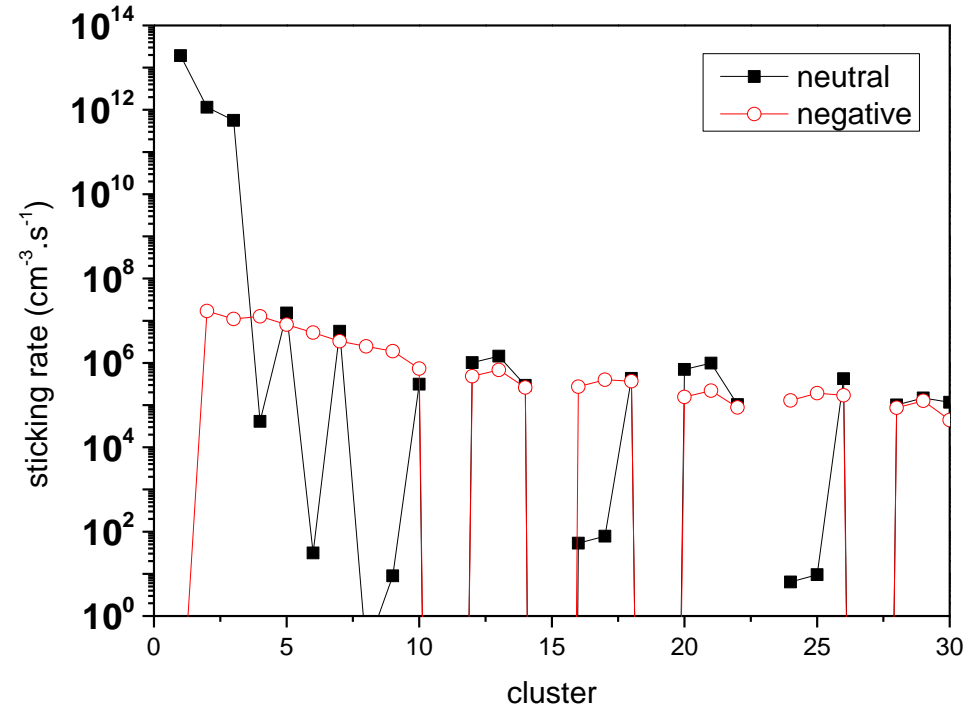

(c)

Figure 8 : Contribution of the different mechanisms involved in the particle aerosol dynamics (a) time variations of the solid particle nucleation and coagulation rate at the field reversal position, i.e., $x=2.3 \mathrm{~cm}$, (b) the number of carbon gained per solid particle and unit time through sticking of carbon cluster at the particle surface and coagulation between solid particle and (c) contributions of the different clusters to the growth of solid particle.

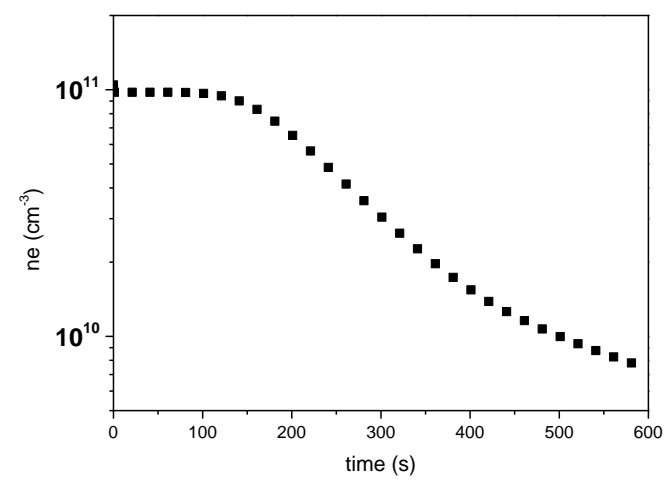

(a)

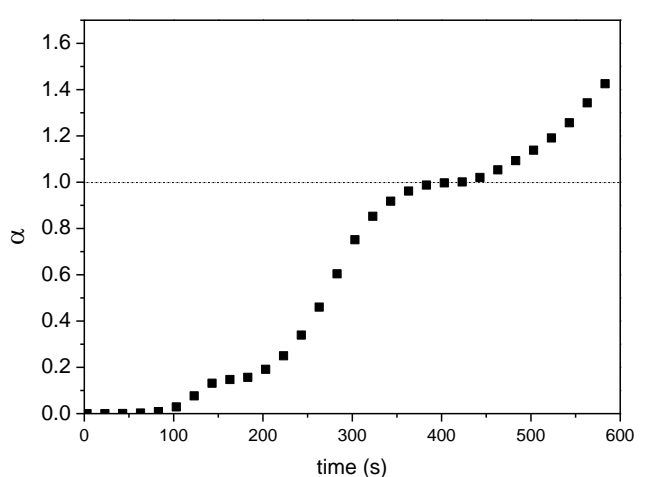

(b)

Figure 9 : Time evolutions of the electron density (a) and electronegativity factor $\alpha(b)$ at the field reversal position, i.e., $x=2.3 \mathrm{~cm}$ 


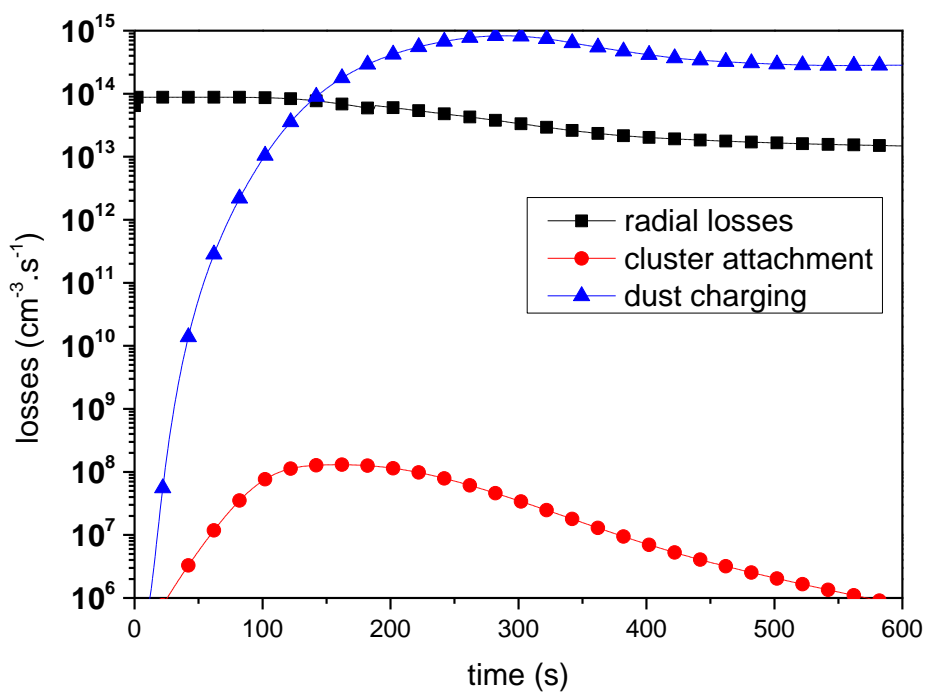

Figure 10 : Time evolutions of the electron loss rates at the field reversal position, i.e., $x=2.3 \mathrm{~cm}, \mathrm{by}$ electron attachment to carbon clusters, electron diffusion to the reactor wall and electron losses by dust particle charging.

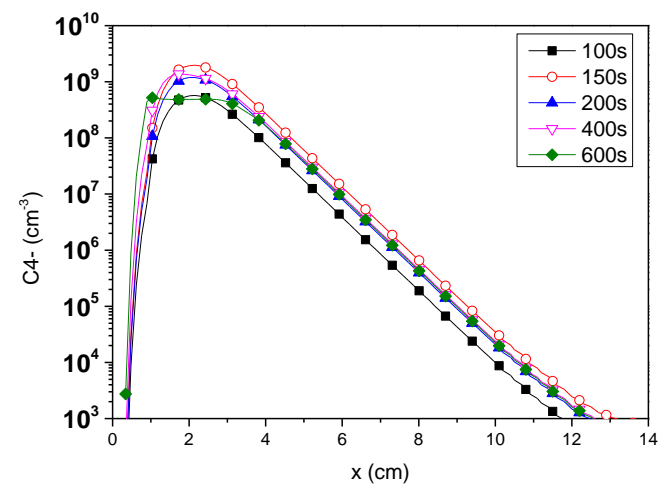

(b)

(c)

Figure 11 : Axial distributions of the $C_{4}{ }^{-}$negative clusters for five time periods : 100, 150, 200,400 and 600 s 


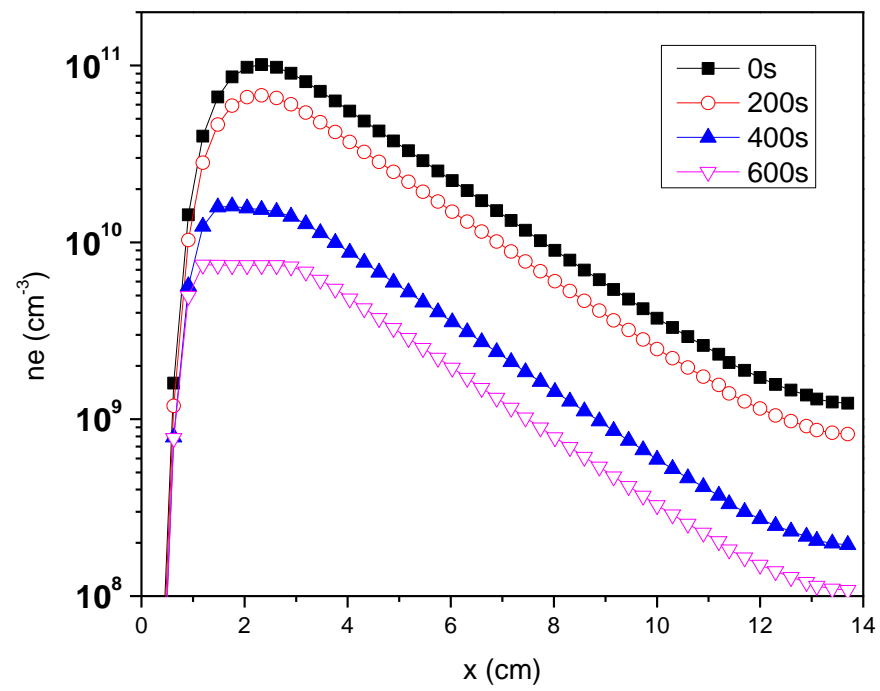

(b)

Figure 12 : Axial distributions of the electron density for five time periods : 100, 150, 200, 400 and $600 \mathrm{~s}$ along the discharge

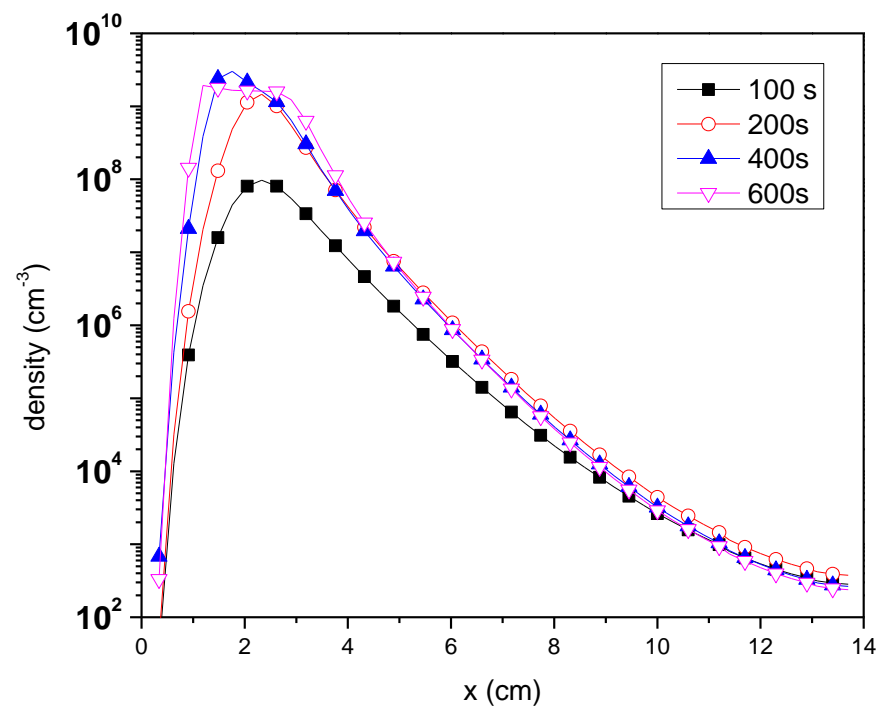

Figure 13 : Axial distributions of the dust particle density for five time periods : 100, 150, 200, 400 and $600 \mathrm{~s}$ along the discharge. 


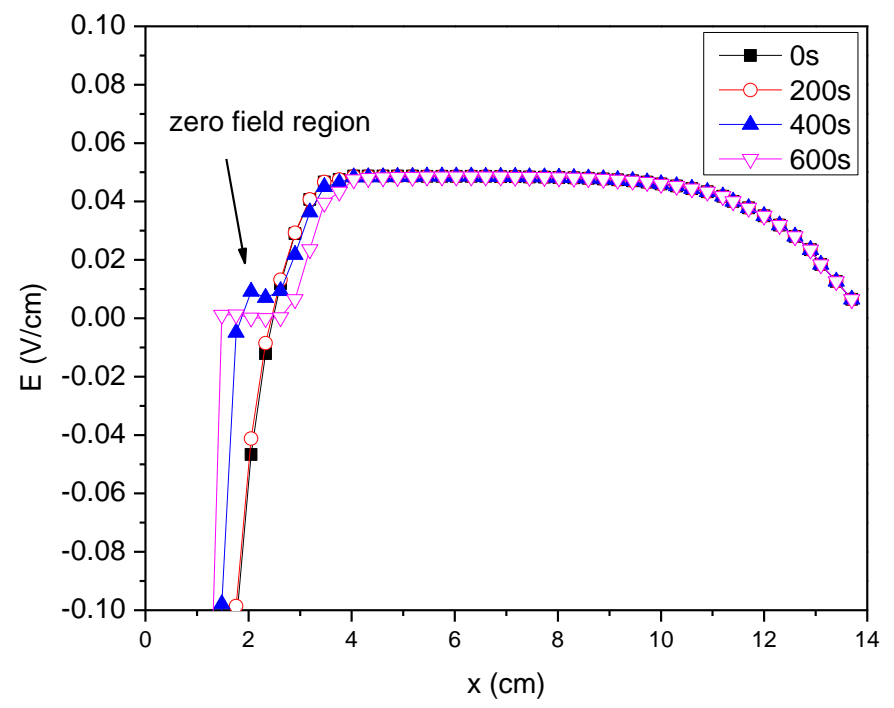

Figure 14: electric field at $0,200,400$ and $600 \mathrm{~s}$ along the discharge.

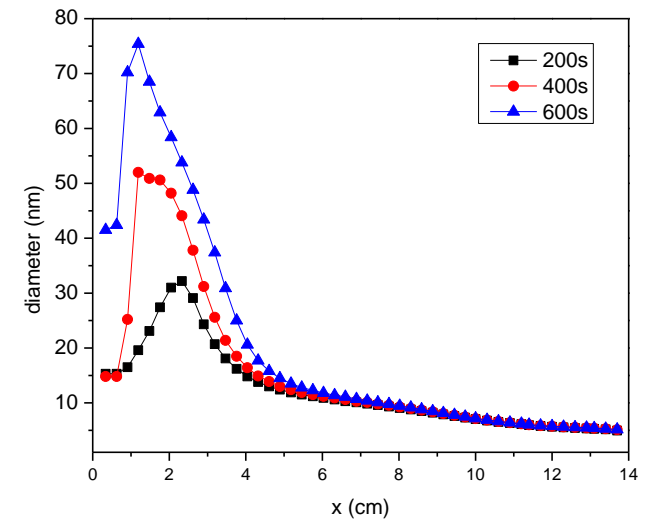

(a)

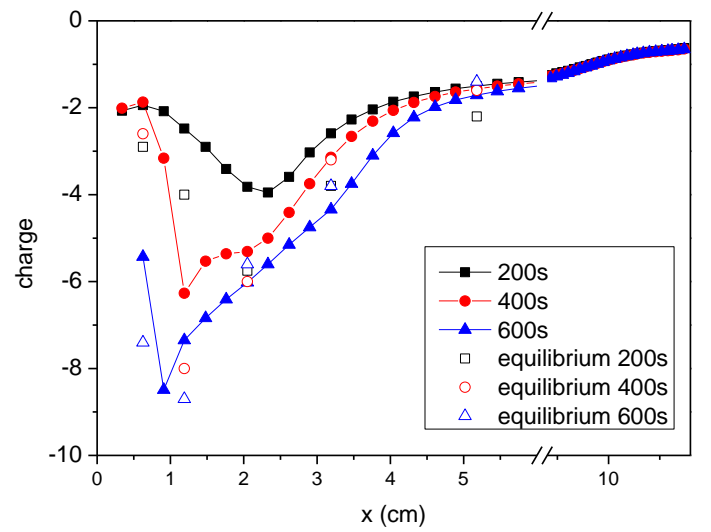

(b)

Figure 15 : Axial distributions of the mean diameter (a) and average charge (b) of the dust particle for five time periods : 100, 150, 200, 400 and $600 \mathrm{~s}$. The charge value obtained from OML theory are also shown (open symbols) 


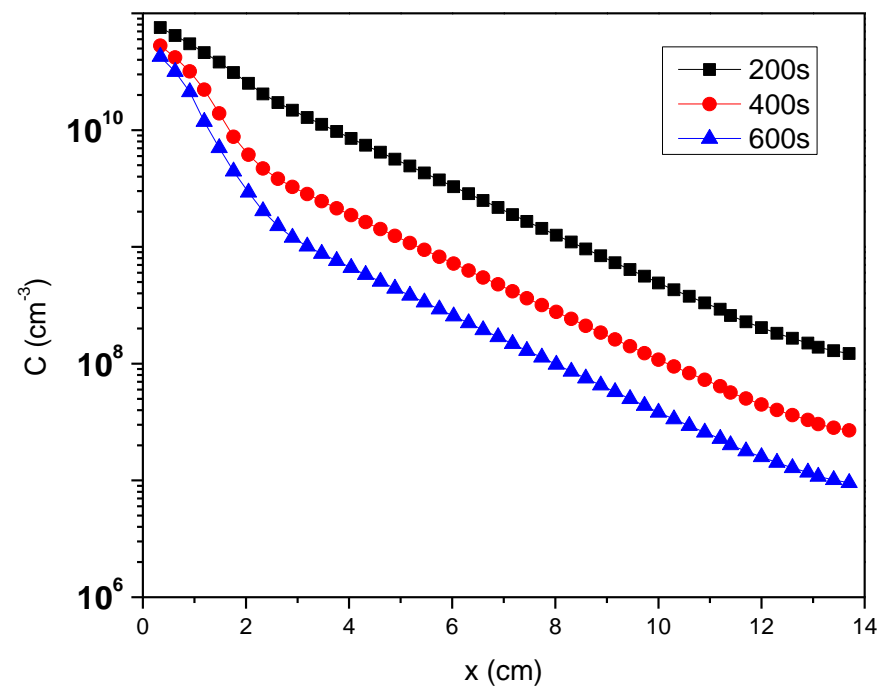

Figure 16: Density axial distributions for the neutral carbon clusters emitted at the cathode: $\mathrm{C}$

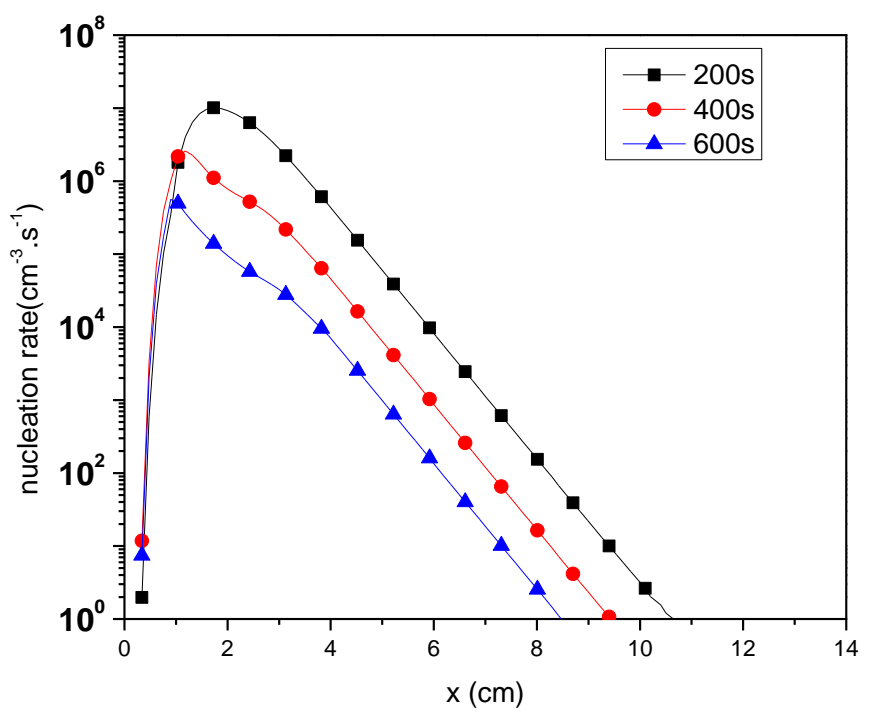

(a) 


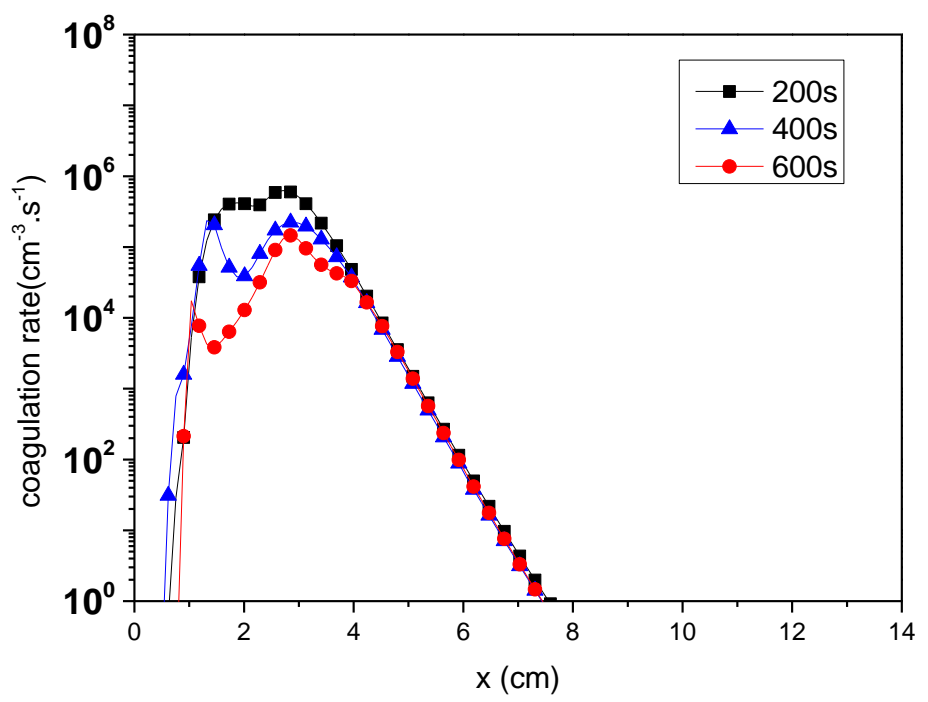

(b)

Figure 17: Axial distributions of the rate of change in dust density through nucleation (a) and coagulation (b)

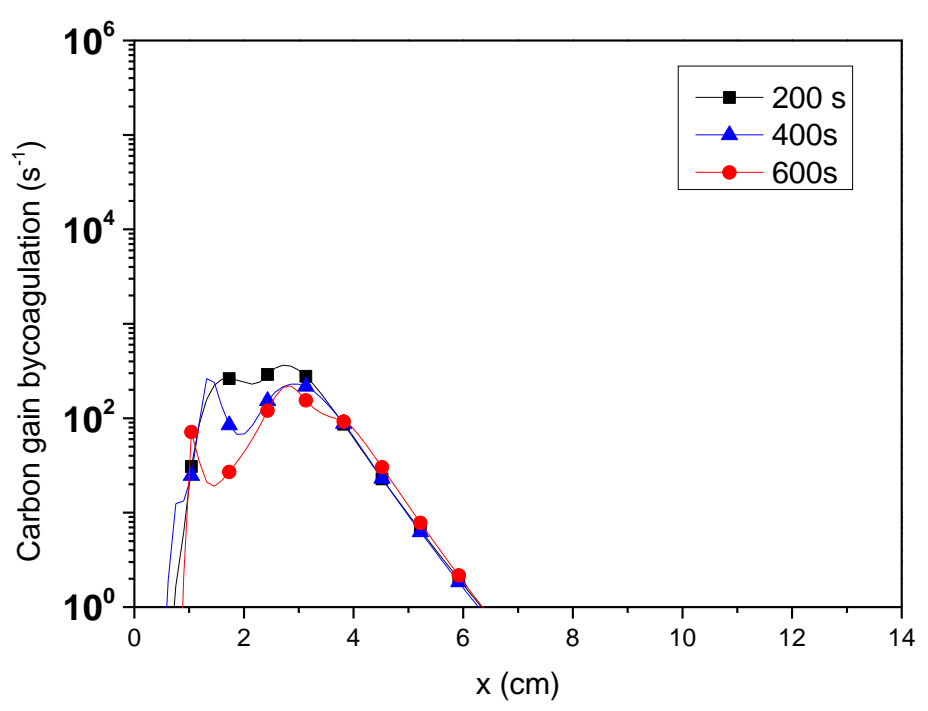

(a) 


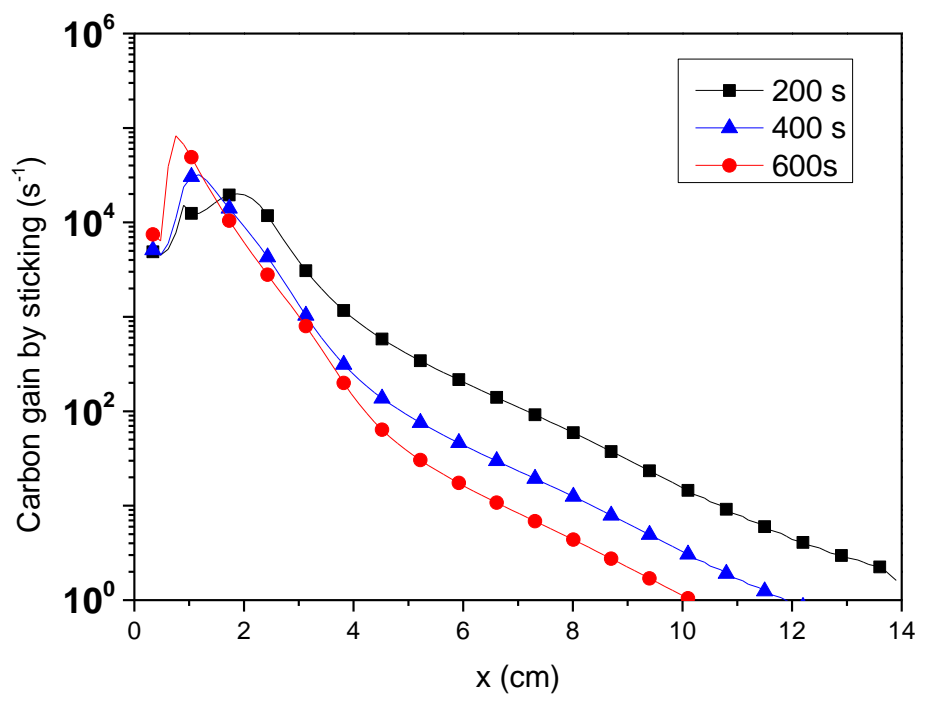

(b)

Figure 18: Axial distributions of the number of carbon gained per dust particle by coagulation (a) and through surface deposition of carbon clusters (b) . 


\section{References}

[1] G. M. Jellum and D. B. Graves, "Particulates in aluminum sputtering discharges," Journal of Applied Physics, vol. 67, pp. 6490-6496, 1990.

[2] S. J. Choi, M. J. McCaughey, T. J. Sommerer, and M. J. Kushner, "Perturbation of the cathode fall in direct-current glow discharges by particulate contamination," Applied Physics Letters, vol. 59, pp. 3102-3104, 1991.

[3] J. P. Boeuf, "Characteristics of a dusty nonthermal plasma from a particle-in-cell Monte Carlo simulation," Physical Review A, vol. 46, p. 7910, 1992.

[4] K. De Bleecker, A. Bogaerts, and W. Goedheer, "Detailed modeling of hydrocarbon nanoparticle nucleation in acetylene discharges," Physical Review E, vol. 73, p. 026405, 2006.

[5] C. Dominique and C. Arnas, "Cathode sputtering and the resulting formation of carbon nanometer-size dust," Journal of Applied Physics, vol. 101, pp. 123304-8, 2007.

[6] C. Arnas, A. Michau, G. Lombardi, L. Couedel, and K. K. Kumar, "Effects of the growth and the charge of carbon nanoparticles on direct current discharges," Physics of Plasmas, vol. 20, Jan 2013.

[7] A. Michau, G. Lombardi, L. Colina Delacqua, M. Redolfi, C. Arnas, P. Jestin, X. Bonnin, and K. Hassouni, "Field Reversal and Particle Growth in DC Discharge," Plasma Chemistry and Plasma Processing, pp. 1-20, 2012.

[8] A. Michau, G. Lombardi, C. Arnas, L. Colina Delacqua, M. Redolfi, X. Bonnin, and K. Hassouni, "Modelling of dust grain formation in a low-temperature plasma reactor used for simulating parasitic discharges expected under tokamak divertor domes," Plasma Sources Science and Technology, vol. 19, p. 034023, 2010.

[9] A. Michau, G. Lombardi, L. Colina Delacqua, R. M., A. C., B. X., and H. K., "Modeling carbonaceous particle formation in an argon graphite cathode dc discharge," Plasma Physics and Controlled Fusion, vol. 52, p. 124014, 2010.

[10] A. Zeinert, C. Arnas, C. Dominique, and A. Mouberi, "Optical properties of carbonaceous nanoparticles produced in sputtering discharges," Journal of Vacuum Science \&amp; Technology A, vol. 26, pp. 14501454, 2008.

[11] Y. P. Raizer, Gas Discharge Physics: Springer, 1991.

[12] L. D. Tsendin, "Electron kinetics in non-uniform glow discharge plasmas," Plasma Sources Science and Technology, vol. 4, pp. 200-211, 1995.

[13] E. Oyarzabal, R. P. Doerner, M. Shimada, and G. R. Tynan, "Carbon atom and cluster sputtering under low-energy noble gas plasma bombardment," Journal of Applied Physics, vol. 104, pp. 043305-12, 2008.

[14] V. A. Schweigert, A. L. Alexandrov, Y. N. Morokov, and V. M. Bedanov, "MINDO/3 study of the interaction of small carbon clusters," Chemical Physics Letters, vol. 238, pp. 110-115, 1995.

[15] P. Agarwal and S. L. Girshick, "Sectional modeling of nanoparticle size and charge distributions in dusty plasmas," Plasma Sources Science and Technology, vol. 21, p. 055023, 2012.

[16] L. Ravi and S. L. Girshick, "Coagulation of nanoparticles in a plasma," Physical Review E, vol. 79, p. 026408, 2009.

[17] K. Hassouni, F. Mohasseb, F. Bénédic, G. Lombardi, and A. Gicquel, "Formation of soot particles in $\mathrm{Ar} / \mathrm{H} 2 / \mathrm{CH} 4$ microwave discharges during nanocrystalline diamond deposition: A modeling approach," Pure and applied chemistry, vol. 78, pp. 1127-1146, 2006.

[18] V. I. Kolobov and L. D. Tsendin, "Analytic model of the cathode region of a short glow discharge in light gases," Physical Review A, vol. 46, p. 7837, 1992.

[19] B. Walch, M. Horányi, and S. Robertson, "Charging of Dust Grains in Plasma with Energetic Electrons," Physical Review Letters, vol. 75, p. 838, 1995.

[20] J. E. Allen, "Probe theory - the orbital motion approach," Physica Scripta, vol. 45, p. 497, 1992.

[21] B. M. Annaratone and et al., "Agglomeration of mesoscopic particles in plasma," New Journal of Physics, vol. 11, p. 103013, 2009.

[22] T. Matsoukas and M. Russell, "Particle charging in low-pressure plasmas," Journal of Applied Physics, vol. 77, pp. 4285-4292, 1995.

[23] M. Olevanov, Y. Mankelevich, and T. Rakhimova, "Coagulation rate of dust grains in a low-temperature plasma," Technical Physics, vol. 48, pp. 1270-1279, 2003.

[24] M. A. Lieberman and A. J. Lichtenberg, Principles of plasma discharges and materials processing. New York: John Wiley \& Sons, 1994. 
[25] J. P. Boeuf and L. C. Pitchford, "Field reversal in the negative glow of a DC glow discharge," Journal of Physics D: Applied Physics, vol. 28, pp. 2083-2088, 1995.

[26] E. A. DenHartog, T. R. O Brian, and J. E. Lawler, "Electron temperature and density diagnostics in a helium glow discharge," Physical review letters, vol. 62, pp. 1500-1503, 1989.

[27] C. Dominique, "Etude d'une décharge à pulvérisation cathodique pour la production de poussières carbonées et étude de la croissance des nanoparticules produites," Université de Provence, 2006.

[28] Bouchoule, Dusty plasmas: physics, chemistry \& technological impacts in plasma processing John Wiley and Sons Ltd, 1999. 